\title{
The genome of the extremophile Artemia provides insight into strategies to cope with extreme environments
}

Stephanie De Vos ${ }^{1,2}$ (D) Stephane Rombauts ${ }^{2} \mathbb{D}$, Louis Coussement ${ }^{3}$, Wannes Dermauw ${ }^{4} \mathbb{D}$, Marnik Vuylsteke ${ }^{5}$, Patrick Sorgeloos ${ }^{1}$ (D) James S. Clegg ${ }^{6}$, Ziro Nambu7 , Filip Van Nieuwerburgh ${ }^{8}$ (D), Parisa Norouzitallab ${ }^{1,9}$ (D), Thomas Van Leeuwen ${ }^{4}$, Tim De Meyer ${ }^{3}$ (D), Gilbert Van Stappen ${ }^{1}$ (D), Yves Van de Peer ${ }^{2,10}$ (D) and Peter Bossier ${ }^{*^{*}}$ (D)

\begin{abstract}
Background: Brine shrimp Artemia have an unequalled ability to endure extreme salinity and complete anoxia. This study aims to elucidate its strategies to cope with these stressors.

Results and discussion: Here, we present the genome of an inbred A. franciscana Kellogg, 1906. We identified 21,828 genes of which, under high salinity, 674 genes and under anoxia, 900 genes were differentially expressed (42\%, respectively $30 \%$ were annotated). Under high salinity, relevant stress genes and pathways included several Heat Shock Protein and Leaf Embryogenesis Abundant genes, as well as the trehalose metabolism. In addition, based on differential gene expression analysis, it can be hypothesized that a high oxidative stress response and endocytosis/exocytosis are potential salt management strategies, in addition to the expression of major facilitator superfamily genes responsible for transmembrane ion transport. Under anoxia, genes involved in mitochondrial function, mTOR signalling and autophagy were differentially expressed. Both high salt and anoxia enhanced degradation of erroneous proteins and protein chaperoning. Compared with other branchiopod genomes, Artemia had $0.03 \%$ contracted and $6 \%$ expanded orthogroups, in which 14\% of the genes were differentially expressed under high salinity or anoxia. One phospholipase D gene family, shown to be important in plant stress response, was uniquely present in both extremophiles Artemia and the tardigrade Hypsibius dujardini, yet not differentially expressed under the described experimental conditions.
\end{abstract}

Conclusions: A relatively complete genome of Artemia was assembled, annotated and analysed, facilitating research on its extremophile features, and providing a reference sequence for crustacean research.

Keywords: Arthropod, Genome, Transcriptome, Assembly, Annotation, Extremophile, Salinity, Anoxia, Artemia, Brine shrimp

\footnotetext{
* Correspondence: peter.bossier@ugent.be

${ }^{1}$ Laboratory of Aquaculture \& Artemia Reference Center, Department of

Animal Sciences and Aquatic Ecology, Faculty of Bioscience Engineering, Ghent University, Ghent, Belgium

Full list of author information is available at the end of the article
}

(C) The Author(s). 2021 Open Access This article is licensed under a Creative Commons Attribution 4.0 International License, which permits use, sharing, adaptation, distribution and reproduction in any medium or format, as long as you give appropriate credit to the original author(s) and the source, provide a link to the Creative Commons licence, and indicate if changes were made. The images or other third party material in this article are included in the article's Creative Commons licence, unless indicated otherwise in a credit line to the material. If material is not included in the article's Creative Commons licence and your intended use is not permitted by statutory regulation or exceeds the permitted use, you will need to obtain permission directly from the copyright holder. To view a copy of this licence, visit http://creativecommons.org/licenses/by/4.0/ The Creative Commons Public Domain Dedication waiver (http://creativecommons.org/publicdomain/zero/1.0/) applies to the data made available in this article, unless otherwise stated in a credit line to the data. 


\section{Background}

Brine shrimp of the genus Artemia are small planktonic crustaceans found in hypersaline environments worldwide [1]. Their larvae (nauplii) are the most commonly used live larval food for marine fish and shellfish species reared in aquaculture $[2,3]$. Artemia comprises six sexually reproducing, diploid species (A. franciscana, A. persimilis, $A$. salina, A. sinica, A. tibetiana, A. urmiana [4]) and several obligate parthenogenetic strains consisting of different clones and ploidies that cannot be grouped under one species [5]. Within each species, numerous local strains exist.

Halophiles are a subtype of extremophile organisms thriving in lakes and ponds with a salt concentration of at least $1.7 \%$ [6]. For reference, sea water has an average salinity of $35 \mathrm{~g} / \mathrm{L}$ [7]. Until now, genomic research on halophiles has been limited to microbial organisms and a few eukaryotes, such as fungi, algae and land plants [8-11]. Because halophilic organisms possess stable enzymes that function under high salinity, efforts are ongoing to discover and utilize halophiles and their enzymes for biotechnology, including biofuel applications [10]. Little is known about the molecular mechanisms specific to animal halophiles. Artemia is one of the few known animal halophiles and is even considered an extreme halophile, tolerating salinities over $300 \mathrm{~g} / \mathrm{L}$ [12]. Being obligate continuous filter feeders, they must excrete excess salt using specialised, developmental-stage-specific organs in nauplii (the salt gland), juveniles (the thoracic epipod) [13], and in adults (gut epithelium and the exopodite segments of the thoracopods) $[14,15]$.

Brine shrimps follow a typical life cycle. Under optimal conditions, adult females produce free-swimming instar I larvae (ovoviviparity) becoming adults within 2-4 weeks, whereas under stress (e.g., high salinity, low oxygen levels), they produce encysted gastrula embryos, named cysts (oviparity), that enter into diapause. These cysts remain viable for years, similar to plant seeds [16]. Cyst diapause is terminated only by strain-specific environmental stimuli (e.g., dehydration, freezing, exposure to low oxygen levels or light), leading to a quiescent state, the latter only terminated by hydration in oxygenated, temperate low salinity water (e.g. $30 \mathrm{~g} / \mathrm{L}$ ) and following a light trigger initiating the hatching metabolism. Hatched larvae grow into adults within 2-4 weeks.

Encysted embryos have a unique tolerance for anoxia, high doses of UV and ionizing radiation, thermal extremes, high and low atmospheric pressure (they hatch and develop in orbital spaceflight) [17] and desiccation $[4,18,19]$ more than any other animal [19-21], partly owing to the presence of biological glasses [21] that contain trehalose and to late embryogenesis abundant (LEA) protein accumulation [22, 23]. Dry brine shrimp cysts are probably one of the most stress-resistant stages of all animals, outliving even tardigrades under ultra-high pressure [24]. Moreover, hydrated cysts have the ability to tolerate continuous, complete anoxia for up to 4 years, at physiological temperatures. The overall metabolism of anoxic embryos is brought to a reversible standstill, including the transduction of free energy and the turnover of macromolecules. Such an extraordinary stability is partly achieved in the cyst by deposition of massive levels of a small heat shock protein (p26) that acts as a molecular chaperone [25].

Knowledge of genes underlying these extreme Artemia phenotypes is of utmost interest and could contribute to making Artemia a promising model for stress response studies or host-microbial interaction studies. Some known examples of such genes are the osmoregulation gene anterior pharynx-defective 1 [26], the cell cycle arrest termination gene ribosomal s6 kinase with direct applications in cancer treatment research [27], and genes coding for Artemia LEA proteins, enhancing desiccation tolerance in mammalian cells, thus enabling engineering of biostable dried cells [28]. However, published genomic resources for $A$. franciscana have been limited to the 15,822 bp mitochondrial genome sequence [29-31], an AFLP-based genetic map [32] and wholetranscriptome studies [33], including a whole transcriptome assembly [34].

Since the publication of the Daphnia pulex genome in 2011 [35], the first of now 46 sequenced and assembled crustacean genomes (Additional file 1: Assembly characteristics of all assembled crustacean genomes), only seven branchiopod genomes have been published. Here, a relatively complete, yet still fragmented genome assembly of $A$. franciscana is presented, representing a largely complete genic portion of the genome. The genome is used to describe the molecular pathways underlying some of the highly unique biology of Artemia, such as its salt and anoxia tolerance.

\section{Results}

\section{Genome assembly and gene annotation}

Illumina and PacBio DNA sequencing reads of an inbred A. franciscana resulted in a total average genome coverage of $53 \mathrm{X}$ by Illumina data (326 million Nextseq paired-end $150 \mathrm{bp}$ reads and 1 million Miseq mate-pair 300 bp reads) and $11 \mathrm{X}$ by PacBio data $(1,809,000$ reads of on average $5531 \mathrm{bp}$ ). The genomic PacBio long reads and paired-end transcript data were used for genome scaffolding. After further gap-closing with the pairedend genomic data, a brine shrimp genome assembly of $849 \mathrm{Mb}$ (see Additional file 2: Evolution of Artemia assembly quality metrics throughout the assembly steps) was obtained, thus achieving a $91 \%$ genome assembly completeness, compared to the $0.93 \mathrm{~Gb}$ Artemia genome size, as estimated by flow-cytometry [36]. Most currently available crustacean genomes are smaller than their 
estimated genome size, which is often ascribed to repeat contraction, or in some cases, mis-estimation of genome size (see Additional file 1). This includes the highquality chromosome level genome assemblies of Daphnia magna and Daphnia carinata (assembly sizes are 42 and $51 \%$ of the respective genome sizes, see Additional file 1). The Artemia genome contains 26,057 contigs, further scaffolded into 20,887 scaffold genome fragments. The longest fragment in the assembly is $855 \mathrm{~Kb}$ long and the assembly shows a GC content of $34 \%$, consistent with earlier estimations of $32 \%$ [37] and a more recent whole-genome estimation of 35\% [36]. The N50 of the Artemia genome (scaffold N50: $112 \mathrm{~Kb}$ ) was below the median N50 of crustacean genomes of similar genome sizes around $1 \mathrm{~Gb}$ (Additional file 1).

To support gene prediction, 15 million RNAseq read pairs from different mixed life stages of the inbred Artemia (whole body) were generated and assembled into a transcriptome (GC 37\%; transcript number 76,045), with values similar to a previously published non-inbred Artemia transcriptome (GC 36\%; transcript number 64,972) [34].

In the Artemia genome, gene structures were predicted with software packages EuGene and Augustus [38, 39], based on integrated data sets of Artemia RNAseq, EST and protein as well as arthropod RefSeq. 21,828 genes were identified and classified as non-repeat associated loci. Remarkably, genes were composed of, on average 4.41 exons per gene, with an intron length of $3458 \mathrm{bp}$, values standing out from other crustaceans. The set of high-confidence protein-coding gene loci was functionally annotated based on homology within NrProt, Genome Ontology (GO) and protein domains. A BUSCO analysis was applied to estimate the genome completeness (Additional file 3: BUSCO results for the A. franciscana genome assembly and annotation), suggesting that $75.5 \%$ of the 1066 BUSCO reference genes were present in the current annotation. The BUSCO reference genes are presumed to be generally present in arthropods, but are known to be biased towards model organisms (insects) [40], with crustaceans being underrepresented. This partially explains the missing BUSCOs.

None of the fragments in the assembly represented the complete mitochondrial genome (Additional file 4: BLAST results for mitochondrial genes in the Artemia genome). Since Artemia cannot be grown axenically to adulthood, some genomic fragments were identified as contaminants and removed from the Artemia genome (Additional file 5: Taxonomic groups of alien genomes identified in the Artemia genome): mainly bacteria (FCB group, Proteobacteria, Terrabacteria, unclassified bacteria), but also eukaryotes (Alveolata, Opisthokonta, Viridiplantae).

\section{Characterization of functional genome and repeat content}

The structural content of the Artemia genome was compared to other crustacean species (Fig. 1a). The A. franciscana genome showed $2.0 \%$ exonic space, similar to Litopenaeus vannamei (3\%), while introns made up 29\% of the genome, exceeding other arthropod genomes shown in Fig. 1, except Eulimnadia texana (30\%). The relatively larger intron size can in part be attributed to the longer PacBio reads included in these genome assemblies [41]. Nevertheless, the intron content of Artemia was more than twice that of L. vannamei, which has a genome size twice that of Artemia.

Use of PacBio reads in the Artemia assembly process allowed to close and identify more repeats, as has been previously noted for other species [41]. Softwares RepeatMasker and RepeatModeler [42, 43] found a repeat content of 58\% (Fig. 1b) in Artemia, higher than in E. texana and L. vannamei, in fact higher than in any other branchiopod or any crustacean. Interspersed repeats (SINEs, LINEs, LTR elements, DNA elements and unclassified interspersed repeats) spanned $53.6 \%$ of the Artemia genome. In addition, small RNAs represented $0.7 \%$ of the Artemia genome.

\section{Comparative genomics}

To identify differences and similarities between the genome of Artemia from the order Anostraca and that of other branchiopod species from distinct taxonomic orders (Cladocera: D. pulex, Notostraca: L. arcticus and Spinicaudata: E. texana) a total of 16,912 orthogroups (gene families shared by different species) were considered in Artemia for expansion and contraction as compared to each studied species (more details in Methods section 2 , Table 1 ).

In total, 10,892 orthogroups were present in at least one branchiopod (Additional file 6: Expanded or contracted Artemia orthogroups compared to other Branchiopoda), of which $0.03 \%$ were contracted and $6 \%$ were expanded in Artemia compared with other branchiopods (Fig. 2). Of the genes in these orthogroups, $14 \%$ were differentially expressed under high salinity or anoxia (See Section "Functional genomics: salt and anoxia tolerance-specific genes"). The most enriched (Fisher's exact test, FDR $\leq 0.05)$ gene ontology classes $(\mathrm{GOs})$ in expanded or contracted gene families in Artemia compared to other Branchiopoda included amongst others (Fig. 3, Additional file 7: GO enrichment of Artemia compared to other Branchiopoda): redox maintenance, transport (metal ions, inorganic cations), protein metabolism and ubiquitination, protein folding and stability, cellular homeostasis, protein (de)phosphorylation, stress responses and finally, nuclear-encoded genes for mitochondrion organization, for cellular (dis)assembly or 


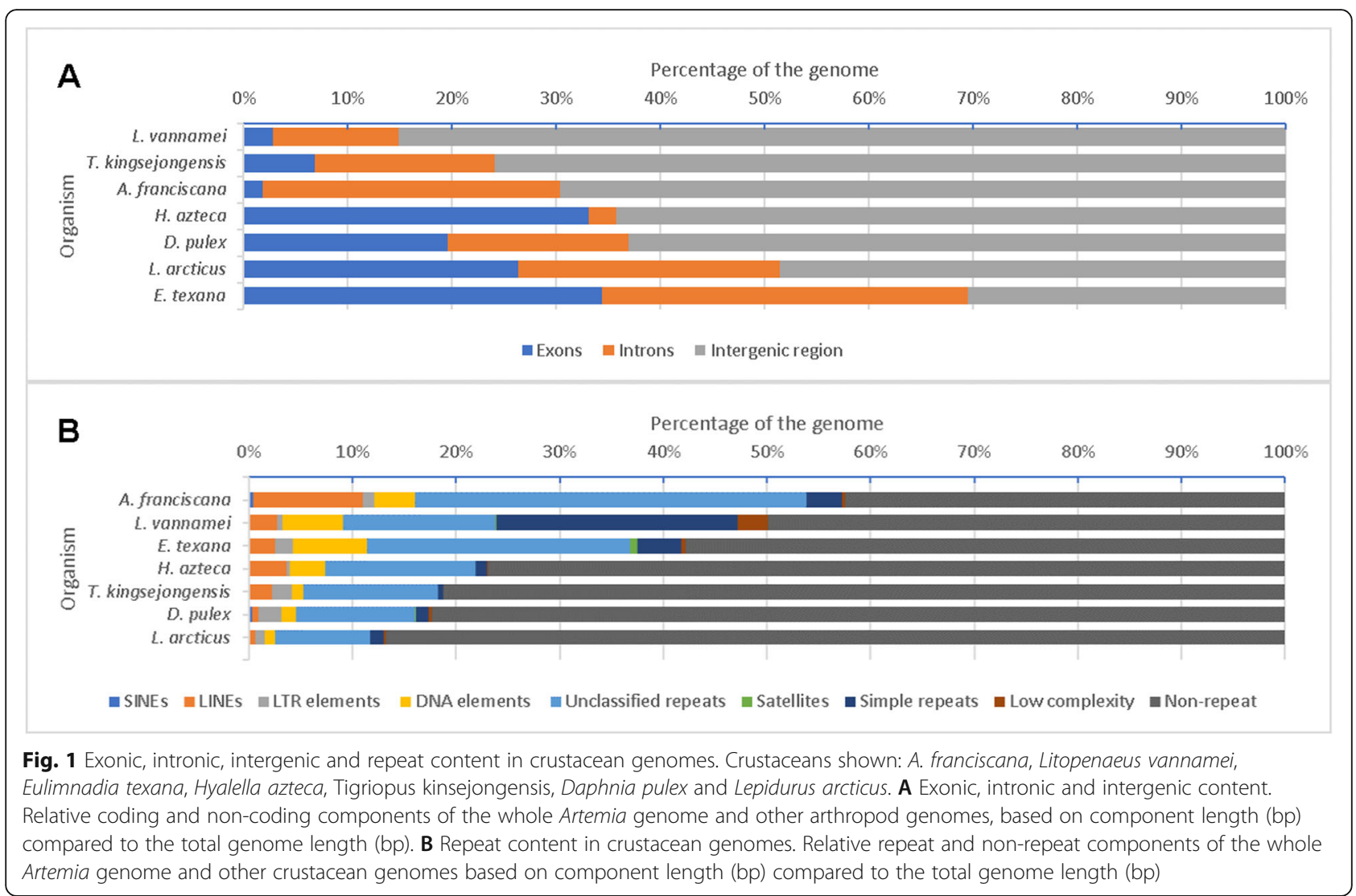

arrangement of the mitochondrion organelle. Many of these processes are involved in either salt or oxygen stress (See Section "Functional genomics: salt and anoxia tolerance-specific genes"). Several orthogroups, including Cytochrome b5-like heme/steroid binding domain superfamily (OG0011697) and the Phospholipase D family (PLD) (OG0009549) were uniquely present in Artemia and also in the extremophile Hypsibius dujardini, a member of the tardigrade phylum (Additional file 8:
Expanded or contracted Artemia and $H$. dujardini orthogroups compared to other Arthropoda) and absent in other branchiopods.

\section{Salt and anoxia tolerance-specific genes Differential expression analyses}

RNAseq data from non-inbred Artemia was sampled and analysed under four different conditions: high salinity $(200 \mathrm{~g} / \mathrm{L})$, low salinity $(30 \mathrm{~g} / \mathrm{L})$, anoxia and normal

Table 1 Species included in comparative genomics analysis

\begin{tabular}{lll}
\hline Species & Taxonomic group & Proteome source \\
\hline Artemia franciscana & Crustacea: Branchiopoda: Anostraca & This study \\
Daphnia pulex & Crustacea: Branchiopoda: Cladocera & {$[44]$} \\
Lepidurus arcticus & Crustacea: Branchiopoda: Notostraca & {$[45]$} \\
Eulimnadia texana & Crustacea: Branchiopoda: Spinicaudata & {$[46]$} \\
Tigriopus kingsejongensis & Crustacea: Copepoda & {$[47]$} \\
Litopenaeus vannamei & Crustacea: Decapoda & {$[48]$} \\
Hyalella azteca & Crustacea: Malacostraca: Amphipoda & {$[49]$} \\
Hypsibius dujardini & Tardigrada & {$[50]$} \\
Drosophila melanogaster & Insecta & {$[51]$} \\
Bombyx mori & Insecta & {$[52]$} \\
Tetranychus urticae & Chelicerata & {$[53]$}
\end{tabular}




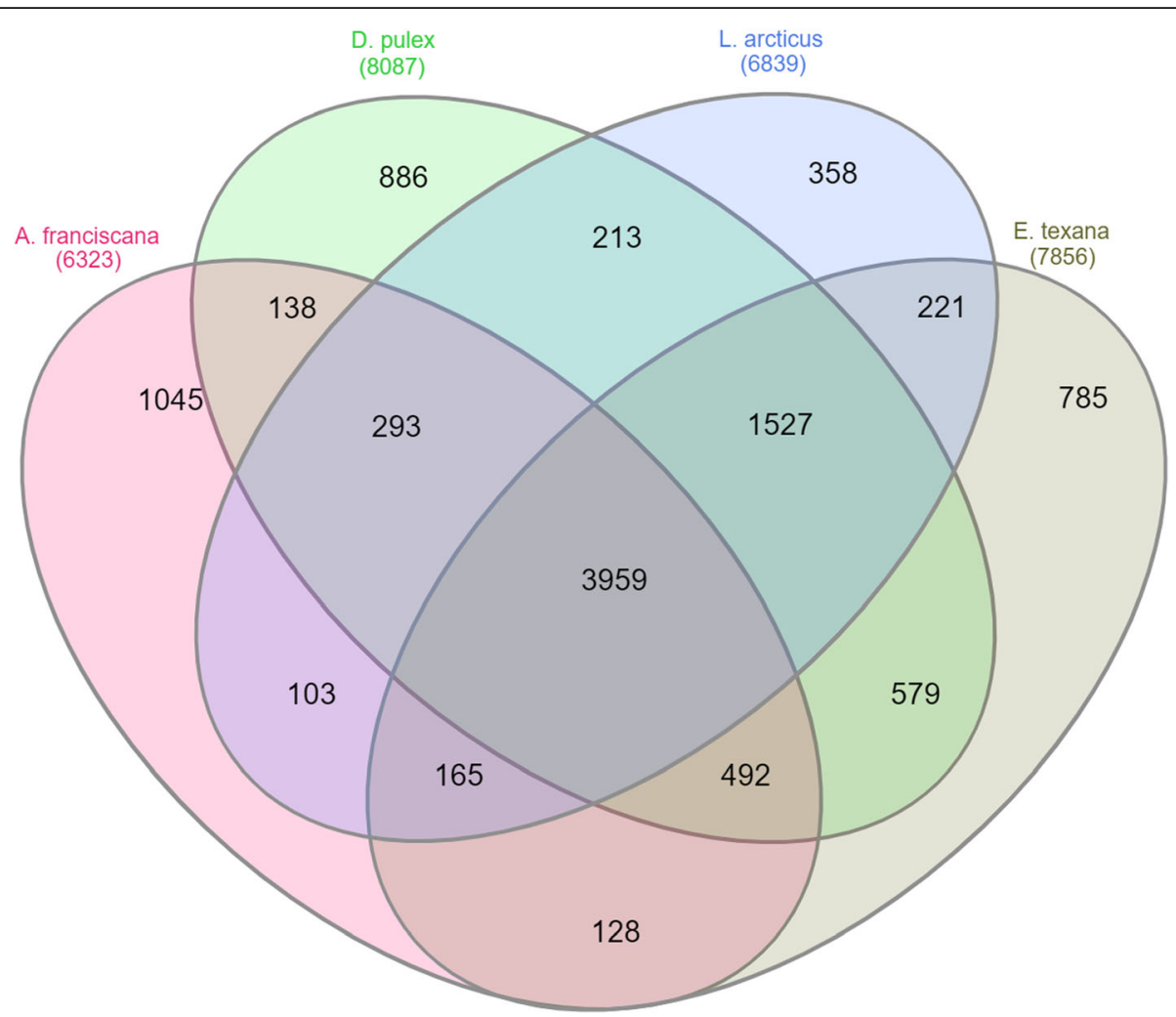

Fig. 2 Comparative genomics between Artemia and other branchiopods. Venn diagram showing the number of orthogroups for each of the branchiopods A. franciscana, Daphnia pulex, Lepidurus arcticus and Eulimnadia texana

oxygen levels. Gene expression of nauplii was compared under high (treatment) and low salinity (control) and gene expression of cysts was compared under anoxia (treatment) and normal oxygen conditions (control). For a detailed overview of the treatments, see Table 2, Methods section 3.2.

Sequence alignment with STAR resulted in an average of $50.3 \%$ uniquely mapped and $35.3 \%$ multimapped reads. This relatively low degree of uniquely mapped reads most likely reflects high sequence variability due to the use of non-inbred Artemia samples. Additionally, the inbred Artemia genome contained relatively short genes, possibly explaining the multimapped reads (Additional file 9: STAR mapping statistics for differential expression analysis in Artemia). As samples from different batches showed different mapping percentages (average difference 14\%, $p=2.5 \mathrm{E}-13$ ) and samples had been randomized over batches, we adjusted for batch effects as a factor during differential expression analysis (see Methods section 3). After data summary, on average $21.3 \%$ of mapped reads overlapped with annotated genes (Additional file 10: Summarization statistics for differential expression analysis in Artemia). Nevertheless, the transcriptome was sufficiently covered to perform differential expression analysis (19X; standard deviation $=7 \mathrm{X})$.
In nauplii under high salinity (compared to low salinity), on average over all time points (15, 30, 45 and $60 \mathrm{~min}$ ), 674 genes were significantly differentially expressed (adj. $\mathrm{PVal}<0.05$ ), of which 459 were upregulated and 215 downregulated.

In cysts under anoxia (compared to cysts with normal oxygen levels), 900 genes were significantly differentially expressed (adj. PVal<0.05), of which 293 were upregulated and 607 downregulated.

The top 20 differentially expressed genes (DEG) with functional annotation, sorted by absolute log fold change (LogFC) under high salinity and anoxia can be found, including the adjusted $P$ value (Adj. Pval) in Tables 3 and 4, respectively (full tables can be found in Additional file 11: Differentially expressed genes under high salinity and Additional file 12: Differentially expressed genes in Artemia under anoxia, respectively).

To identify processes and pathways involved in salt and oxygen stress responses, significantly enriched GOs (Fisher's exact test FDR $\leq 0.05$ ) and pathways (Fisher's exact test corrected for multiple testing, FDR $\leq 0.05$ ) were determined in the list of significantly differentially expressed genes $(p<0.05)$ with software packages OmicsBox (Biobam, GUI software previously known as Blast2GO) and STRING v11.0, respectively. Only significantly overrepresented GOs and pathways (from here on more simply referred to as 'enriched GOs' or 'enriched 


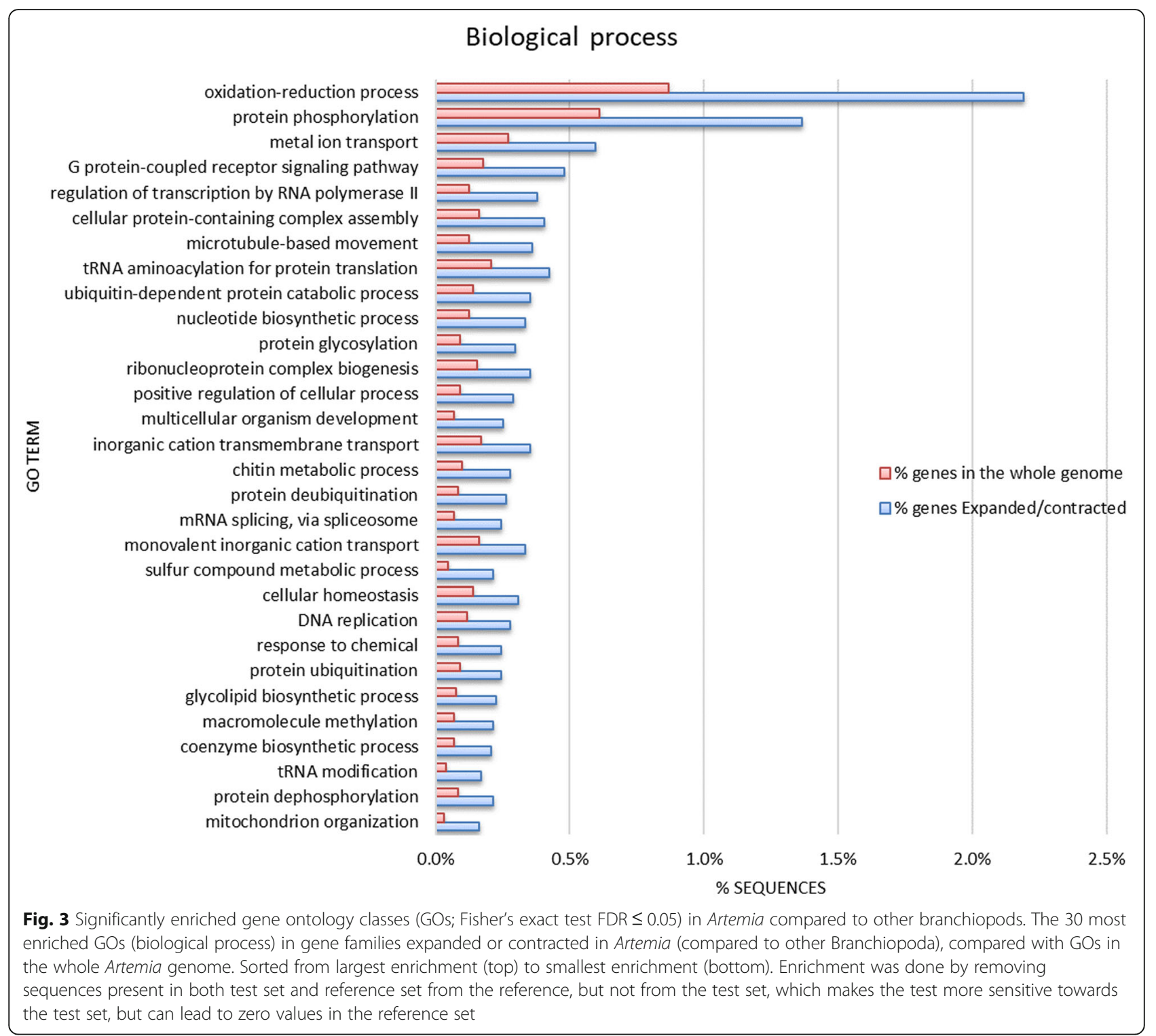

pathways') obtained through these exploratory analyses are mentioned in the results and discussion sections.

\section{Genes, GOs and pathways associated with high salinity}

Under high salinity, several GOs for biological processes, molecular functions and cellular components were significantly enriched (FDR $\leq 0.05$; Fig. 4; Additional file 13:
GO enrichment in Artemia under high salinity) and this was also the case for several pathways (FDR $\leq 0.05$; Additional file 14: Pathway enrichment in Artemia under high salinity). To facilitate further analysis, significant DEG, GO and pathway enrichment results under high salinity (Additional files 11, 13 and 14) were summarized into one file (Additional file 15).

Table 2 Artemia sampling for RNAseq, including sampling time after treatment start and ARC cyst bank ID

\begin{tabular}{|c|c|c|c|c|c|c|}
\hline Treatment & Life cycle stage & Sampling time (min) & Biological replicates per sampling time & Cyst ID & Species & Strain \\
\hline Anoxia & cysts & 60 & 3 & ARC1767 & A. franciscana & SFB \\
\hline Hydration, normal oxygen & cysts & 60 & 3 & ARC1767 & A. franciscana & SFB \\
\hline High salinity (200 g/L) & instar I/II nauplii & $15,30,45,60$ & 3 & ARC1767 & A. franciscana & SFB \\
\hline Low salinity (30 g/L) & instar I/II nauplii & $15,30,45,60$ & 3 & ARC1767 & A. franciscana & SFB \\
\hline
\end{tabular}


Table 3 The top 20 differentially expressed genes (LogFC-based) in A. franciscana under high salinity

\begin{tabular}{|c|c|c|c|}
\hline Gene Id & LogFC & Adj. Pval & Functional annotation \\
\hline $\operatorname{artfr5078g00010~}$ & 1.91 & 1.04E-05 & LEA6 \\
\hline artfr4102g00020 & 1.62 & $6.94 \mathrm{E}-06$ & catalase-like isoform $\times 2$ \\
\hline artfr3493g00020 & 1.59 & $7.82 \mathrm{E}-05$ & major facilitator superfamily domain-containing protein 12-like \\
\hline artfr401g00070 & 1.57 & 1.79E-07 & major facilitator superfamily domain-containing protein 12-like \\
\hline artfr498g00040 & 1.35 & $8.45 \mathrm{E}-05$ & glutamate-cysteine ligase catalytic subunit isoform X3 \\
\hline artfr8416g00010 & 1.26 & $6.09 \mathrm{E}-03$ & trehalose-phosphate synthase 1 \\
\hline artfr328g00050 & -1.24 & 5.80E-04 & ATP-dependent DNA helicase pif1-like \\
\hline artfr363g00050 & 1.23 & $2.25 \mathrm{E}-05$ & MFS-type transporter SLC18B1-like \\
\hline artfr1784g00020 & 1.17 & $1.94 \mathrm{E}-03$ & chorion peroxidase-like \\
\hline artfr1161g00070 & 1.14 & 3.69E-03 & mitochondrial group 1 LEA protein precursor \\
\hline artfr4423g00020 & 1.12 & 2.83E-04 & cysteine proteinase precursor \\
\hline artfr1761g00010 & 1.10 & $2.10 \mathrm{E}-03$ & organic cation transporter protein-like \\
\hline artfr6563g00010 & 1.08 & 1.77E-03 & patatin-like phospholipase domain-containing protein 2-like \\
\hline $\operatorname{artfr1273g00010}$ & 1.07 & $1.12 \mathrm{E}-04$ & cyclin-Y \\
\hline artfr537g00010 & 1.07 & $1.24 \mathrm{E}-05$ & catalase homolog A \\
\hline artfr92g00010 & 1.07 & 3.85E-05 & LEA6 \\
\hline artfr1351g00060 & 1.05 & 4.28E-09 & kruppel homolog 1 \\
\hline artfr328g00060 & -1.05 & 3.63E-03 & ATP-dependent DNA helicase pfh1-like \\
\hline artfr175g00080 & 1.05 & $6.58 \mathrm{E}-06$ & dolichyl pyrophosphate Man9G/cNAc2 alpha-1,3-glucosyltransferase-like \\
\hline artfr8387g00020 & 1.04 & $1.03 \mathrm{E}-02$ & Senescence-specific cysteine protease SAG39 \\
\hline
\end{tabular}

Table 4 The top 20 differentially expressed genes (LogFC-based) in A. franciscana under anoxia

\begin{tabular}{|c|c|c|c|}
\hline Gene Id & LogFC & Adj. Pval & Functional annotation \\
\hline artfr1563g00010 & -2.86 & $1.42 \mathrm{E}-07$ & TBC1 domain family member 23 \\
\hline artfr690g00020 & -2.78 & $6.92 \mathrm{E}-07$ & putative DBH-like monooxygenase protein 2 \\
\hline artfr1292g00020 & -2.67 & $1.62 \mathrm{E}-08$ & protein peritrophin-1-like \\
\hline artfr2964g00030 & 1.94 & 4.17E-06 & $\mathrm{N}$-lysine methyltransferase SETD8-like \\
\hline $\operatorname{artfr} 2663 g 00020$ & 1.90 & $8.72 \mathrm{E}-07$ & lysozyme 1-like \\
\hline artfr2034g00020 & 1.88 & 1.10E-07 & pentafunctional AROM polypeptide-like \\
\hline $\operatorname{artfr} 427 g 00100$ & 1.83 & $3.16 \mathrm{E}-06$ & splicing factor PTSR1, putative \\
\hline artfr1672g00030 & 1.80 & $3.38 \mathrm{E}-06$ & $A B C$ protein, subfamily $A B C H$ \\
\hline artfr3907g00020 & 1.72 & 8.70E-05 & protein bark beetle \\
\hline artfr503g00010 & 1.64 & $1.16 \mathrm{E}-07$ & cytochrome P450 CYP18A1 \\
\hline artfr1202g00050 & -1.60 & $3.49 \mathrm{E}-03$ & transcription initiation factor TFIID subunit 4 \\
\hline artfr2964g00010 & 1.59 & 1.13E-08 & integumentary mucin A.1-like \\
\hline artfr188g00020 & 1.59 & $7.50 \mathrm{E}-14$ & cuticle protein \\
\hline artfr1482g00010 & 1.59 & $2.21 \mathrm{E}-08$ & aminopeptidase YwaD \\
\hline artfr316g00060 & 1.56 & $1.28 \mathrm{E}-14$ & serine proteinase stubble \\
\hline artfr61g00120 & 1.51 & $3.16 \mathrm{E}-06$ & adenosine monophosphate-protein transferase Fic \\
\hline artfr1404g00010 & -1.49 & $5.42 \mathrm{E}-03$ & heme-binding protein 2-like \\
\hline artfr10544g00020 & -1.48 & 1.19E-02 & Beadex/dlmo protein \\
\hline artfr1487g00020 & 1.48 & $1.10 \mathrm{E}-04$ & Cdk1/cks1 \\
\hline artfr2018g00030 & 1.48 & 4.19E-05 & clathrin coat assembly protein \\
\hline
\end{tabular}




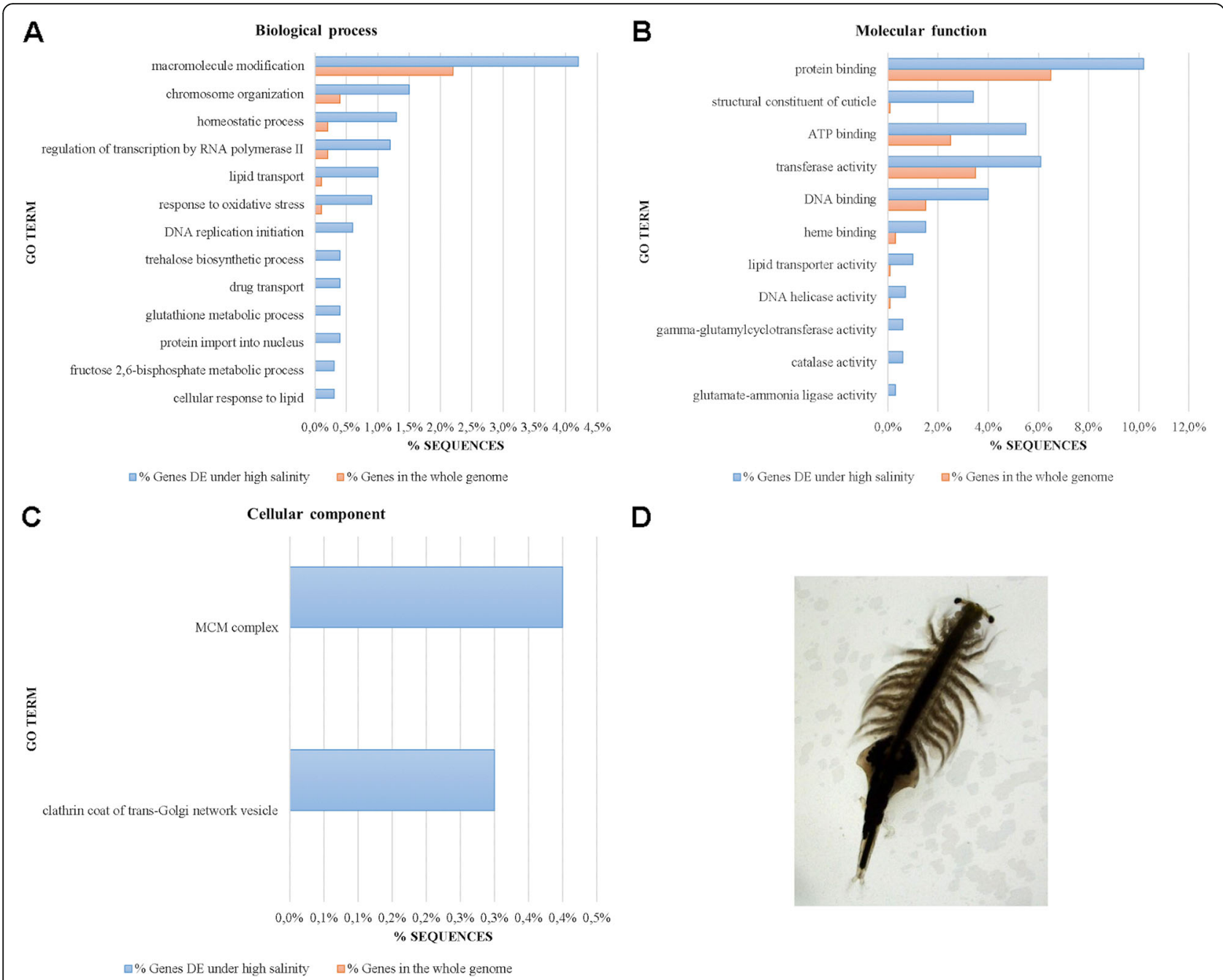

Fig. 4 Significantly enriched gene ontologies (Fisher's exact test, FDR $\leq 0.05$ ) in the differentially expressed gene list under high salinity in Artemia. Biological processes (A), molecular functions (B) and cellular components (C) for genes differentially expressed under high salinity are shown. GO terms are sorted from the largest (top of the graph) to the smallest difference (bottom of the graph) between \% sequences in the whole genome and in DE genes. Enrichment was done by removing sequences present in both test set and reference set from the reference only, but not from the test set, which makes the test more sensitive towards the test set, but can lead to zero values in the reference set. $\mathbf{D}$ Binocular image of an adult female $A$. franciscana

DNA metabolism Interestingly, Artemia under salt stress displayed significant downregulation of DNA repair genes (nucleotide excision repair protein, ERCC-8, ERCC-5, DNA methyltransferase 1 associated protein 1, see Additional file 15). Additionally, genes responsible for telomere maintenance were significantly and highly downregulated, including ATP-dependent DNA helicase pif 1-like (Fig. 4b: DNA helicase activity, Table 3, Additional file 15), yet it remains to be determined whether this reflects genuine telomere biology or rather relates to the more general process of maintaining genome integrity. This gene was part of the significantly enriched GO "chromosome organisation" which also contained the following upregulated genes: two histone-lysine $\mathrm{N}$ methyltransferases (SETDB1-B, CG1716), potentially involved in epigenetic transcriptional activation/repression, sister chromatid cohesion protein PDS5 potentially also involved in DNA replication inhibition and $R N A$ polymerase-associated protein CTR9 involved in histone modifications (Additional file 15).

In contrast, Artemia displayed significant upregulation of several DNA replication licensing factors mcm3, mcm3-like mcm4, mcm5-like, mcm6, and mcm6-b-like. These genes are responsible for replication stress response [54] and are involved in the significantly enriched "MCM (mini chromosome maintenance) complex" (Fig. 4a, c, Additional file 15). Finally, the pyrimidine metabolism, DNA replication, spliceosome and the RNA transport pathways were also significantly enriched. 
Oxidation-reduction and response to oxidative stress Salt-stressed Artemia displayed a clear redox response, with all involved genes upregulated under salt stress. Significant GO enrichment was present in the processes "response to oxidative stress", overlapping with significant GO enrichment of "catalase activity" with six highly upregulated catalases and a peroxidase (Table 3). Other significantly enriched GOs were the "glutathione metabolic process" with four upregulated glutamate-cysteine ligases and a glutathione-specific gamma-glutamylcyclotransferase as well as the "heme binding" process with upregulated Cytochrome P450 $315 a 1$ and (cyto) globin, a known scavenger of nitric oxide or reactive oxygen species (Fig. 4a, b, Additional file 15).

Clathrin coat of trans-Golgi network vesicle (CCV) In plants, the salt stress response with e.g. classically upregulated chloride ion transporter genes [55] is intensively studied at the genome-wide level, while in animals (fish, crustaceans), fewer studies are available and are mainly focused on lower levels of salt (up to seawater salinity) and on specific gene groups [56]. Endocytosis, a form of active transport in animals and plants, brings substances into the cell by surrounding them with a cell membrane, then budding off inside the cell to form a vesicle containing the ingested material. In plants, salt stress induces rapid clathrin-mediated endocytosis of NADPH oxidases to generate intravesicular reactive oxygen species (ROS) that presumably act as signalling molecules critical for salt stress tolerance [57]. During the first endocytosis steps in plants, clathrin-coated pits are internalized to form clathrin-coated vesicles (CCVs), which assemble at the trans-Golgi network [58]. In salmon eggs exposed to low salt levels $(5 \mathrm{~g} / \mathrm{L})$, genes of the clathrin-mediated endocytosis signalling pathway are activated as well [59]. Under high salinity ( $200 \mathrm{~g} / \mathrm{L})$, Artemia showed significant GO enrichment for CCVs, with upregulated clathrin heavy chain genes (Fig. 4c, Additional file 15). Clathrin is the major protein of the polyhedral coat of CCV's.

\section{Protein processing in the endoplasmic reticulum and} chaperones Molecular chaperones, many of which are heat-shock proteins (hsps), are an important class of molecules with various functions under stress. In Artemia and throughout the animal and plant kingdoms, they are a pivotal part of stress response [60]. The pathway for protein processing in the endoplasmic reticulum (KEGG pathway shown in Fig. 5) was significantly enriched and included upregulation of genes responsible for protein recognition by luminal chaperones (nef, grp94), recognition and targeting of terminally misfolded proteins (EDEM, PDIs), transport of misfolded proteins to the cytosol (TRAM, $p 97)$ and ER-associated protein degradation assisted by several heat shock proteins (hsp40, hsp70 hsp90). Correctly folded proteins were likely transported by Golgi bodies, the formation of which is assisted by a protein transport protein ( $\operatorname{Sec} 13$ / 31).

Other chaperone genes were found significantly upregulated as well $($ FDR $<0.05)$ : four different Hsp22/hsp20like chaperones, heat shock protein 213 (with Alpha crystallin domain), four different DnaJ genes, a HSC70-like protein and two heat shock protein 90 like proteins (Additional file 15).

LEA proteins So far, LEA proteins have been known as osmotic stress or desiccation proteins [61]. Expression of three groups of late-embryogenesis abundant (LEA) genes has been found in Artemia: groups 1, 3, and 6 [62]. In this study, eight LEA genes were significantly $(\mathrm{p}<0.05)$ upregulated under high salinity (See Additional file 11: Differentially expressed genes in Artemia under high salinity): 1) Five group 6 LEA genes, of which two were among the top 20 DEG (see Table 3), one of which (artfr92g00010) has been described before [62], 2) two putative group 1 LEA genes, one currently still broken in three parts, and 3) one group 3 LEA gene. In total, nine different LEA genes can be found in the Artemia genome, all of which are significantly upregulated under high salinity, except one (artfr2598g00030). While the current study shows that an important subset of the LEA genes in the Artemia genome is significantly upregulated under acute salt stress, aside from their previously known expression under desiccation conditions, [62] their potential function under other physiological conditions remains to be determined.

Metabolic pathways Several genes involved in the trehalose metabolism were significantly upregulated: trehalose-phosphate synthase 1, responsible for the first step in trehalose synthesis, facilitated trehalose transporter Tret1-like, a transporter of trehalose, and a trehalase-like gene, probably catalysing the conversion of trehalose into glucose and glycerol.

Remaining significantly enriched pathways were the biosynthesis pathway of amino acids, including cysteine and methionine. The carbon metabolism pathway was also significantly enriched (KEGG pathway shown in Additional file 16), including the citrate cycle, and the metabolism of glyoxylates, dicarboxylates, nucleotide sugars, fructose and mannose.

An overview of all significantly enriched pathways can be found in Additional file 14 (FDR $\leq 0.05)$.

Additionally, salt stress significantly upregulated four major facilitator superfamily genes (Additional file 15), two of which were among the top 20 differentially expressed genes (Table 3). Some major facilitator genes described in plants are involved in small molecule 


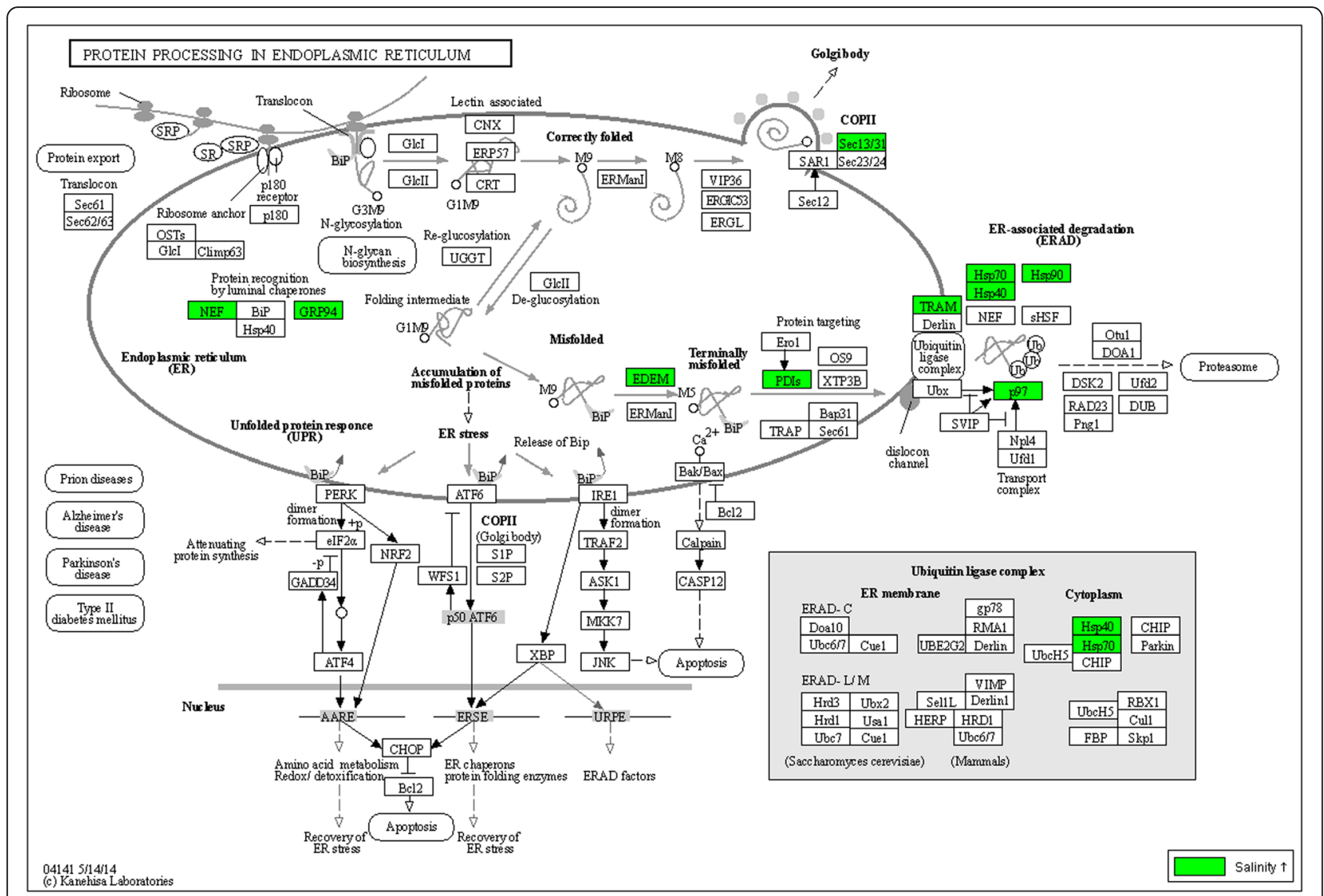

Fig. 5 The pathway "protein processing in the endoplasmic reticulum". This pathway is significantly enriched (Fisher's exact test corrected for multiple testing, FDR $\leq 0.05)$ in the differentially expressed gene list in Artemia under high salinity. Significantly upregulated genes $(p<0.05)$ in Artemia are indicated on the Daphnia pulex pathway map dpx04141 from the KEGG database. No genes of this pathway were significantly downregulated in Artemia. Gene names and Artemia gene IDs are linked in Additional file 14 (tab sheets 1 and 2)

transport, with gene deletions responsible for increased sensitivity to salt [63]. Hence, it can be hypothesized that major facilitator superfamily genes are involved in coping with the sharp salt increase from 30 to $200 \mathrm{~g} / \mathrm{L}$. The latter remains to be substantiated by establishing their physiological role with for instance RNAi experiments on Artemia, a methodology which can currently be used reliably in Artemia [64].

Interestingly, of the 832 DEG under high salinity in Artemia, 481 could not be ascribed to a GO class, indicating that some of the mechanisms involved remain to be unravelled.

\section{Genes, GOs and pathways associated with anoxia}

Under anoxia, several gene ontology classes for biological processes, molecular functions and cellular components were significantly enriched (FDR $\leq 0.05$; Fig. 6 , Additional file 17: GO enrichment in Artemia under anoxia) and this was also the case for several pathways (FDR $\leq 0.05$; Additional file 18: Pathway enrichment in Artemia under anoxia). To facilitate further analysis, significant DEG, GO and pathway enrichment results under anoxia (Additional files 12, 17 and 18) were summarized into one file (Additional file 19).

Mitochondrial function Under anoxia, many genes involving mitochondrial function were significantly upregulated, indicating that enhancement of the mitochondrial metabolism may be involved in coping with anoxic circumstances: 1) genes responsible for mitochondrial protein translation (leucine--tRNA ligases), also part of the significantly enriched GO "leucine-tRNA ligase activity" (Fig. 6b, Additional file 19); 2) eight genes, present in the significantly enriched cellular component GO "mitochondrial envelope" (Fig. 6c, Additional file 19): genes respectively responsible for Cytochrome $\mathrm{C}$ oxidase assembly and maturation (Cytochrome c oxidase assembly protein PET191, protein SCO1 homolog), a gene required for mitochondrial cristae morphogenesis (MSF1 protein), a mitochondrial intermembrane chaperone (Tim13-B), a mitochondrial outer membrane import complex protein (Metaxin-2), a 


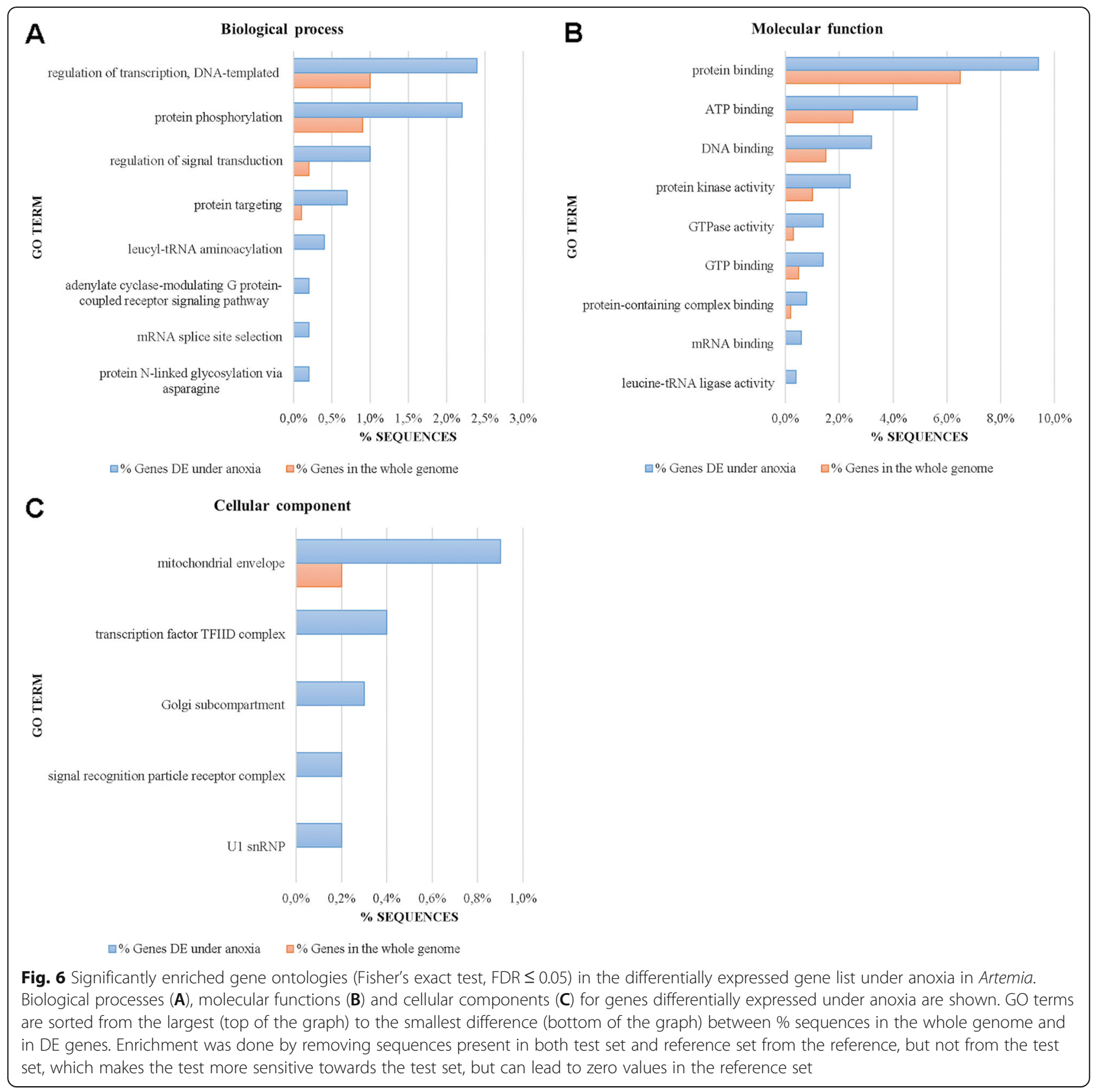

gene responsible for ATP production in the mitochondrial membrane (ATP synthase epsilon chain) and a proton pump in the mitochondrial envelope (vacuolar $\mathrm{H}[+$ Jvacuolar $H+$-ATPase); 3) six different mitochondrially coded genes (28S ribosomal protein S35, 39 S ribosomal protein L19, 39 S ribosomal protein L2, $39 S$ ribosomal protein L22, mitochondrial, $39 S$ ribosomal protein L27, $39 S$ ribosomal protein L37).

mTOR, the phagosome and autophagy Hypoxia is known in humans to activate autophagy and to inhibit the $m T O R$ gene, which in its turn further activates autophagy [65]. The mTOR signalling pathway was significantly enriched in Artemia under anoxia with 10 DEG (Additional file 19). The phagosome is responsible for tissue homeostasis. Under stress, it potentially functions as an autophagosome to degrade (damaged) cytosolic organelles for nutrients by fusing with lysosomes containing digestive enzymes. Under anoxia in Artemia, the phagosome pathway was significantly enriched (Additional file 19, KEGG pathway shown in Fig. 7), with gene sets partly overlapping with the significantly enriched autophagy pathway (Additional file 19). 


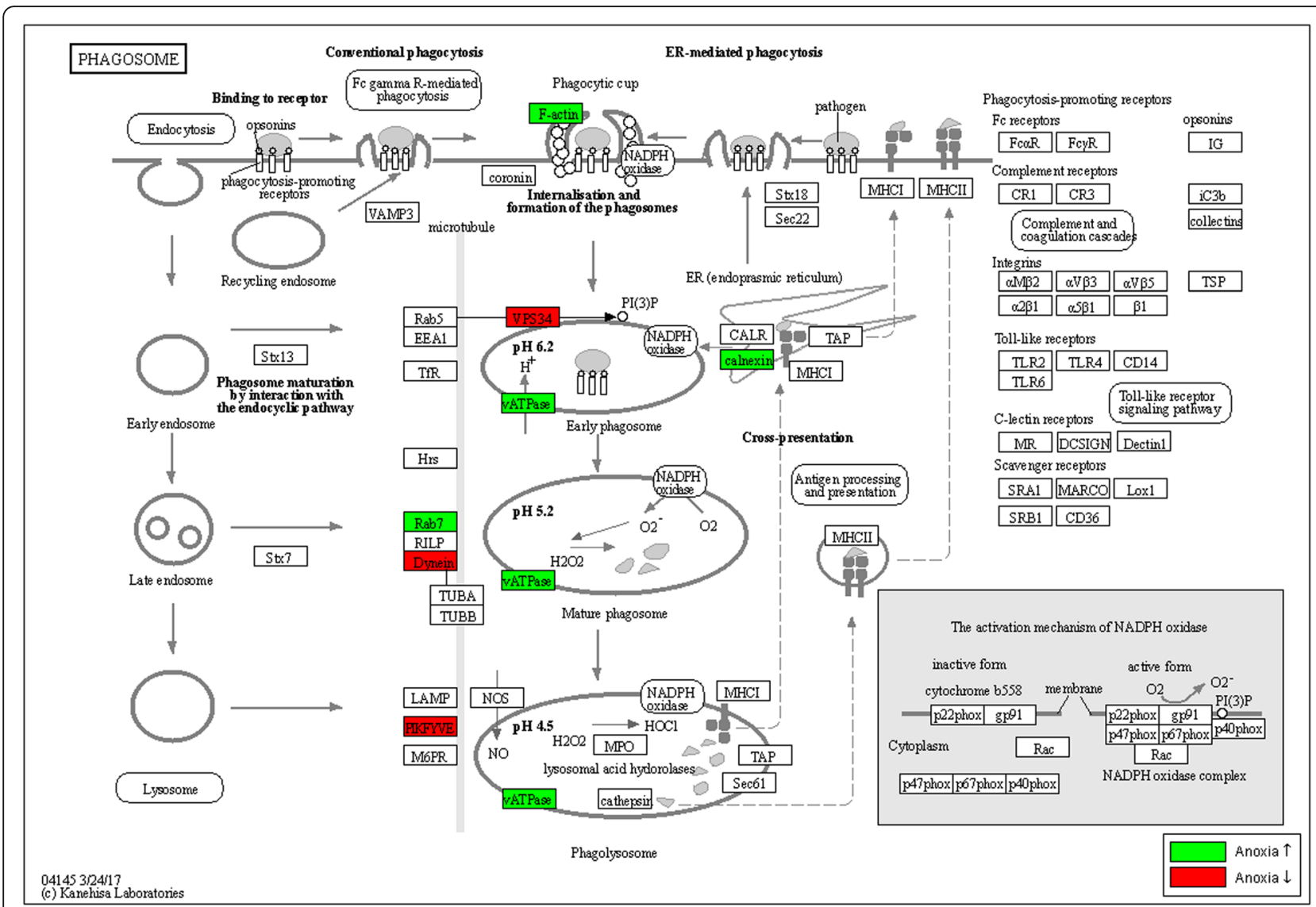

Fig. 7 The pathway "Phagosome". This pathway is significantly enriched (Fisher's exact test corrected for multiple testing, FDR $\leq 0.05)$ in the differentially expressed gene list in Artemia under anoxia. Significantly up- and downregulated genes $(p<0.05)$ in Artemia are indicated on the Daphnia pulex pathway map dpx04145 from the KEGG database. Gene names and Artemia gene IDs are linked in Additional file 18 (tab sheets 1 and 2)

Degradation of erroneous mRNA and proteins The "mRNA surveillance" pathway, a quality control mechanism that detects and degrades abnormal mRNAs, was significantly enriched under anoxia in Artemia and contained only upregulated DEGs (Additional file 19).

The "N-glycan biosynthesis" pathway was also significantly enriched within DEGs under anoxia in Artemia (KEGG pathway shown in Additional file 20). N-glycans are critical for proper protein folding and quality control by chaperones in the endoplasmic reticulum (ER), a process now known as the "calnexin/calreticulin cycle" in the unfolded protein response and ER-assisted degradation [66].

As under high salinity, the pathway "protein processing in endoplasmic reticulum", responsible for removing erroneous proteins in the ER, was significantly enriched under anoxia and included amongst others three significantly downregulated $h s p 70$ genes and a significantly upregulated hsc70 gene. Furthermore, several other chaperone genes were significantly upregulated under anoxia: a mitochondrial $10 \mathrm{kDa}$ heat shock protein, known in humans to prevent misfolding and promote refolding of unfolded proteins generated under stress in the mitochondrial matrix, as well as heat shock protein Hsp-12.2 and Dnajc8 (Additional file 19).

DNA repair, transcription, RNA and protein transport and signalling Two DNA repair pathways were significantly enriched in DEG, the "homologous recombination" pathway for reparation of double strand breaks and the "Fanconi anemia" pathway for reparation of interstrand crosslinks (Additional file 19).

In medical science, it is known that long-term hypoxia leads to transcriptional and/or translational downregulation of most DNA repair pathways, including DNA double-strand break repair, mismatch repair, and nucleotide excision repair [67]. This is potentially what happens in Artemia as this experiment measures the effects after an hour of complete anoxia, which is more extreme than a regular hypoxic state.

Finally, several pathways related to signalling, transcription and RNA and protein transport were 
significantly enriched in Artemia under anoxia: the "basal transcription factors" pathway responsible for transcription activation (KEGG pathway shown in Additional file 21), the "RNA transport" pathway containing only upregulated genes, the "spliceosome" and the "protein transport" pathways as well as the "phosphatidylinositol signalling" pathway (Additional file 19).

An overview of all significantly enriched pathways under anoxia is shown in Additional file 18 (FDR $\leq 0.05$ ).

\section{Genes associated with salinity and anoxia}

Only 49 (3\%) of all DEG were differentially expressed under both high salinity and anoxia, with 11 having no functional annotation. There were no common DEG among the top 20 DEG under high salinity and anoxia (Tables 3 and 4, respectively).

\section{Discussion}

This is the first time that an Anostracan genome is assembled. Unique challenges were overcome for assembly and annotation of the Artemia genome. First, sequence variation was high, owing to the diploidy of Artemia and the need for several animals to generate sufficient DNA for long read DNA sequencing. To reduce sequence variation, the initial focus of the genome project was shifted from non-inbred Artemia to an inbred strain. Secondly, the unprecedented $58 \%$ repeat content in the Artemia genome led to a fragmented initial genome assembly. To our knowledge, Artemia has the highest repeat content ever recorded in crustaceans. Assembly fragmentation was greatly reduced on a limited budget by scaffolding the genome with a long-read DNA sequence coverage of $10 \mathrm{X}$ and N50 as well as gene integrity were further improved by scaffolding the genome with RNA sequence data. Thirdly, the Artemia genome had genes with relatively short exons and exceptionally long introns (3458 bp) compared to other arthropods. This was overcome by using tailor-made gene modelling for Artemia. As a result, and in spite of its unprecedented repeat content, unusual gene structure and limited budget, the Artemia genome assembly had a GC content of $34 \%$ in correspondence with literature, a genome assembly contiguity of the same order of magnitude as other similar-sized crustacean genomes (scaffold N50 of $112 \mathrm{~Kb}$ ), and a 21,828 gene genome annotation, containing $75.5 \%$ of the BUSCO reference genes found in other arthropods. While BUSCO analysis results are often not included in crustacean genome publications, this is in a similar range as the $88 \%$ BUSCO reference genes found in the E. texana genome, a highly contiguous crustacean assembly considered as a new standard for crustacean genomes [46].

Artemia has been described as an extremophile, with nauplii and adults able to survive and thrive at extreme salinities in lakes and saltworks and producing cysts able to survive anoxic conditions.

Genome-wide research on halophiles has so far largely been limited to microbial organisms and a few eukaryotes, such as fungi, algae and land plants [8-11]. As far as we know, this study is the first genome-based study of a halophilic animal. Recently, a transcriptome-based differential expression analysis identified salt stress responses in Artemia involving EIF (eukaryotic initiation factor), Heat shock $70 \mathrm{kDa}$ protein cognate, the chitin metabolic pathway, DNA repair, ubiquitination, cell cycle arrest (La-related protein), lipid metabolism and (metal) ion transport [34]. The current study, while genome-based and more extensive, largely confirms the findings of the previous transcriptome analysis: 1) $E I F$ was involved (significant upregulation of genes NAT1A and EIF4AIII), 2) a HSC70-like gene was significantly upregulated, 3) DNA repair-related genes were significantly downregulated, 4) the gene La-related protein $6 A$ was significantly upregulated, and 5) the GO terms "cellular response to lipid", "lipid transporter activity" and "heme binding" as well as the cellular component GO "structural constituent of cuticle" were significantly enriched.

All genes related to "structural constituents of the cuticle", including several cuticle proteins were downregulated in Artemia under high salinity. Under salinities as high as $200 \mathrm{~g} / \mathrm{L}$, growth in Artemia is substantially slowed down due to its increased energy needs for osmoregulation, whereas at lower salinities (e.g. the $30 \mathrm{~g} / \mathrm{L}$ control in our experiment), the brine shrimp larva moults every few hours into a subsequent developmental stage until maturity [68]. Moulting involves many regulatory mechanisms and related genes, including regulation of cuticle-related enzymes such as chitinase and structural proteins of the cuticle [69]. Transcriptomic analysis in crustaceans Portunus trituberculatus [70]; Euphausia superba [71] and L. vannamei [72] at different stages of the moulting cycle showed upregulation of structural constituents of the cuticle during the moulting cycle, with upregulation of cuticle genes during pre-moult and downregulation during inter-moult [12]. Exposure of the Artemia nauplii to high salinity thus corresponds with a prolonged state of inter-moult.

For several biological processes previously reported in plant salt stress, similar processes were found in Artemia and these were significantly enriched in the DEGs under the Artemia salt stress response in this study. In plants, salt stress causes genotoxicity in which DNA alteration/ damage can arise as a consequence of errors in DNA replication and DNA repair [73], which also seems to be the case in Artemia. In plants as in Artemia, MCM family genes show salt stress-responsive behaviour [74] and the phagosome is also an enriched gene ontology feature, as found in the salt-tolerant plant Suaeda salsa under high $(30 \mathrm{~g} / \mathrm{L})$ salinity [11]. 
In halophytic plant species, there is strong evidence in favour of clathrin-dependent vesicular transport mechanisms of $\mathrm{Na}^{+}$and $\mathrm{Cl}^{-}$ions as a feature of salt tolerance. During endocytosis, parts of the plasma membrane enclose extracellular substances in vesicles eventually fusing with lysosomes and vacuoles to isolate the plant from the excess salt. During exocytosis, these vesicles export excess ions via plant salt glands [75]. Interestingly, under high salinity, Artemia showed significant GO enrichment of processes in the clathrin-coated vesicles, including clathrin upregulation. Clathrin was also part of an expanded gene family in Artemia compared to other non-extremophile branchiopods. Hence, it appears possible that Artemia would transport salt via clathrindependent vesicular transport (endocytosis) for final salt excretion (exocytosis). It remains to be established if exocytosis is ongoing via the Artemia salt gland [76], which is still present at the sampling time points chosen in this study and remains present, at least until larval stage instar III, after which it is resorbed in adulthood.

Protein processing in the endoplasmic reticulum and chaperones, both found to be important Artemia salt stress responses in this study, play a key role in targeting and degrading misfolded proteins by ATP-dependent heat shock proteins. Both the use of ATP-dependent HSP and, potentially, endo-and exocytosis of salt, are energy-intensive processes, which may explain the enhanced carbon metabolism in Artemia under high salinity.

Salt-stressed Artemia also showed a redox response similar to that observed in plants [77] and in the intertidal flatworm Macrostomum lignano [78], all perturbed in redox homeostasis under salt stress. Salt stress generally induces reactive oxygen species (ROS), causing oxidative stress-induced damage to cellular components, which can be neutralized by antioxidants [79]. Catalases and peroxidases, heme enzymes involved in ROS metabolism, were upregulated in Artemia under salt stress, suggesting that they were directly responsible for ROS control. Transition metals are known to function as balancing factors and signalling for ROS in plants [80]. Transition metal binding genes were also upregulated under salt stress in Artemia. Salt stress most likely induced a strong oxidative stress in Artemia, which was then balanced with transition metal binding proteins.

LEA proteins were part of an expanded gene family in Artemia compared to other branchiopods (Additional file 6; OG0007033, 9 genes including one with "seed maturation protein" motive). They are important for cellular protection during desiccation in eukaryotic cells by e.g., stabilizing membranes [81] and were upregulated in Artemia under high salinity, potentially to protect cells from desiccation. Such a high number of LEA genes has also been described in tardigrades (with variable numbers depending on the species), where they contribute to desiccation tolerance $[82,83]$. On the other hand, homologs of other tardigrade-specific proteins involved in tardigrade desiccation tolerance (such as CAHS, SAHS, and MAHS) [84], were not present in the Artemia genome (results not shown). Tardigrades and Artemia thus share some common strategies relative to osmotic stress and/or desiccation (e.g., ROS mitigation, trehalose production, HSP induction, LEA), but they probably also harbour species-specific genes.

The genes in the hypoxia-inducible factor (HIF) pathway are known master regulators of oxygen sensitivity and distribution inside cells, and are therefore considered highly conserved in most animal groups. Homologs of the Marsupenaeus japonicus hypoxia-inducible factor (HIF-1 $\alpha$ [85]) were identified in the Artemia genome (results not shown) but were not differentially expressed under anoxia in this study. Homologs of $V H L$ (Von Hippel-Lindau tumor suppressor), regulating HIF- $\alpha$ degradation and $F I H-1$, hydroxylating a specific residue of HIF- $\alpha$ under normoxic conditions, were both absent in the Artemia genome. Within the Crustacea, even if the HIF pathway can be found in several species, it has been lost in other crustaceans, such as the intertidal Tigriopus californicus, where alternative hypoxic stress response mechanisms are believed to play a major role, such as genes involved in cuticle reorganization and ion transport [86]. It is possible that Artemia has partially lost its HIF pathway and uses an alternative hypoxia response through cuticle reorganization and ion transport. This seems plausible, as Artemia is a halophile and the gene cuticle protein was one of the top 20 differentially expressed genes in Artemia under anoxia. Moreover, it could be that the HIF pathway is involved in regulating low-oxygen (hypoxic) conditions, but not full anoxia such as Artemia was exposed to in this study.

In the freshwater crayfish Orconectes virilis under severe and prolonged anoxia (5-20 h), cAMP-dependent protein kinases strongly decrease and phosphatase activity is strongly increased [87], while hypoxia inhibits mTOR, activating autophagy [88]. In Artemia, many protein kinases were up- and downregulated, while most (protein) phosphatase genes were upregulated (Additional file 19). It is possible that increased phosphatase activity plays a role in metabolic adjustment of Artemia to prolonged anoxia. The $m T O R$ signalling and autophagosome pathways were significantly enriched in Artemia under anoxia as well. It seems that under anoxic stress in Artemia, the mTOR pathway activates the autophagosome pathway to recycle (damaged) cytosolic organelles for nutrients.

Mitochondria are major oxygen consumers and a potential source of ROS. In response to hypoxia, the mitochondrial metabolism often becomes more efficient [89]. 
In Artemia, mitochondrial function was clearly enhanced with significant upregulation of genes responsible for ATP production in the mitochondrial membrane, cristae morphogenesis, mitochondrial transport and intermembrane chaperoning.

In this study, comparative genomics showed that Artemia had the highest number of unique gene families compared to other Branchiopods, containing gene expansions in domains important for salt stress tolerance, such as redox response and endocytosis-related mechanisms, as well as for anoxia tolerance, such as protein phosphorylation. The PLD gene family, while its genes were not differentially expressed under high salinity or anoxia in Artemia, was expanded compared to other, non-extremophilic branchiopods. The PLD gene family consists of lipid metabolic genes that degrade membranes, thus triggering a signalling cascade molecule influencing the mTOR pathway towards survival or inflammation responses, thus initiating proliferation, transcription, cytoskeletal organization and NADPH oxidase and vesicle formation for exo- or endocytosis [90]. PLD genes, also found in tardigrades, were first described in plants, in which they are typically activated by a wide variety of stressors: cold-, drought- and salinitystress, wounding, pathogenic elicitation and phosphate starvation [91].

While many mechanisms underlying the salt stress and the anoxia responses of Artemia were identified, still $32 \%$ of DEG under salt stress and $21 \%$ under anoxia had no known function. Since functional gene annotation is based on homology with other organisms, it is possible that mechanisms unique to Artemia are still hidden within these unknown genes.

Artemia has been used in the study of hostmicrobial interactions (e.g. Vibrio infection), uniquely allowing Vibrio challenge tests under gnotobiotic conditions [92]. Artemia is also, through its short generation time and large population size, susceptible to truncation-based genetic selection. Cysts generated in such experiments can be stored and hatched simultaneously, allowing for common garden experiments [93]. Moreover, parthenogenetic strains of Artemia are instrumental in the study of transgenerational phenotype inheritance and hence in the research of epigenetic processes [93]. Finally, Artemia shows a relatively large similarity with the recently published $L$. vannamei genome $(2.45 \mathrm{~Gb}$ [94];), containing over half of its orthogroups, including typical L. vannamei immunity-related genes, without expansion or contraction (results not shown). It is anticipated that with both annotated genomes currently now available, it will be possible to fully explore the potential of Artemia as a model species for commercial aquatic crustaceans in the fields of host-microbial interaction, genetics and epigenetics.

\section{Conclusions}

Here, we present the genome assembly and annotation for an inbred A. franciscana, as well as comparative genomics with other animals and functional genomics under high salinity and anoxia. Compared with other branchiopods, the Artemia genome has many contracted and expanded gene families involved in different strategies for coping with stressors including high salt and anoxia. This means that the differences between Artemia and other branchiopods can probably be largely attributed to the stress coping abilities of Artemia. Under high salinity, Artemia potentially uses plant-like, energy-intensive strategies to enclose excess salt in the cell, as well as DNA repair, extensive molecular chaperoning, redox and osmotic balancing, and LEA proteins and trehalose against desiccation. Under anoxia, mitochondrial function was enhanced, while the mTOR pathway activated the autophagy pathway, potentially for recycling of damaged cell components as nutrients.

\section{Methods}

All bioinformatics up until genome characterization with DBG2OLC were performed on the VIB-PSB Biocomputing Linux cluster, all subsequent steps were performed on the HPC-UGent, supported by the Flemish Supercomputer Center.

\section{Genome assembly Sampling, DNA extraction, sequencing and read processing}

Sampling Inbred A. franciscana Kellogg, 1906 (Great Salt Lake (GSL), Utah, USA) were reared at the Department of Medical Technology, University of Occupational and Environmental Health, Kitakyushu, Japan: a 48 generation inbred female parent (sample \#421, as shown in Nambu et al. under "GSL2" [95]) and a 62 generation inbred male parent (sample \#870, as shown in Nambu et al. under "GSL2" [95]), were crossed to produce $F_{2}$ progeny cyst specimens kept at $4{ }^{\circ} \mathrm{C}$, in $20 \mathrm{~g} / \mathrm{L}$ salt. Cysts of "Specimen 9 " were hatched $\left(28^{\circ} \mathrm{C}\right.$, salinity $\left.10 \mathrm{~g} / \mathrm{L}\right)$, the nauplii were harvested. Considering the hatching time frame, these nauplii should be instar I or II, which are difficult to separate during the hatching process. Nauplii were reared until sexual maturity in aerated seawater with added sea salt (Instant Ocean ${ }^{\circ}$ USA, $28^{\circ} \mathrm{C}$, final salinity $70 \mathrm{~g} / \mathrm{L}$ ) and fed with microalgae Tetraselmis suecica. Mature animals were rinsed with sterile distilled water, the phenotypic sex of each individual offspring was determined visually and females were selected for further processing. Females were favoured since they are the heterogametic sex, thus ensuring sequencing of both the $\mathrm{Z}$ and the $\mathrm{W}$ chromosomes. For gut evacuation before DNA extraction, the females were kept overnight in a cellulose solution $(1.5 \mathrm{~g} / \mathrm{L}$, type 20 , Sigma-Aldrich 
USA) [96], followed by removal of the brood pouch. The animals were stored individually at $-20^{\circ} \mathrm{C}$.

DNA extraction DNA was individually extracted from seven female individuals grown from Specimen 9 cysts according to a modified CTAB-method for shrimp tissue [97]. Briefly: each sample, homogenized in $150 \mu \mathrm{l}$ of CTAB buffer with $3 \mu$ l RNAse A (from the Wizard ${ }^{\circ}$ Genomic DNA Purification Kit, Promega USA) was kept for $30 \mathrm{~min}$ at $37^{\circ} \mathrm{C}$, then re-incubated $30 \mathrm{~min}$ in CTAB buffer $\quad(0.25 \% \quad \mathrm{v} / \mathrm{v} \quad$ 2-mercaptoethanol). After homogenization, $750 \mu \mathrm{l}$ of extra CTAB buffer was added and the mix was left at $25^{\circ} \mathrm{C}$ for $30 \mathrm{~min}$ and PCA solution was added $(600 \mu \mathrm{l}, 25: 24: 1 \mathrm{phenol} / \mathrm{chloroform} /$ isoamylalcohol). After centrifugation, $800 \mu \mathrm{l}$ of the upper aqueous phase was supplemented to $600 \mu \mathrm{l}$ of CA solution (24:1 chloroform/isoamylalcohol) and the mix was homogenized. To $700 \mu \mathrm{l}$ of the upper aqueous phase, $630 \mu \mathrm{l}$ of isopropanol was supplemented. The mix was incubated for $1 \mathrm{~h}$ at $-70{ }^{\circ} \mathrm{C}$. After centrifugation, the pellet was washed with $600 \mu \mathrm{l}$ of ethanol $70 \%$, air-dried in a $60^{\circ} \mathrm{C}$ oven and resuspended in $20 \mu \mathrm{l}$ of sterile distilled water. DNA quality and concentration were assessed on an agarose gel and by NanoDrop ${ }^{\mathrm{Tm}}$ spectrophotometer (Thermo Fisher Scientific USA).

DNA from inbred females served as the template for PacBio (Pacific Biosciences USA) and Illumina (USA) sequencing technologies.

Sequencing and read processing: Illumina DNA from one female individual (sample name F2) from Specimen 9 was sequenced with several technologies, as described below.

One library (500-800 bp) was prepared for the Illumina sequencing: Illumina NextSeq library prep was done with the NEBNext ${ }^{\circ}$ Ultra $^{\text {Tm }}$ DNA Library Prep Kit for Illumina ${ }^{\circ}$ (New England Biolabs, USA). The library was paired-end sequenced with Illumina NextSeq 500 High 300 Medium technology (read length $150 \mathrm{bp}$, intended output 35X, NucleomicsCore, Leuven) and with Illumina MiSeq 600 v3 technology (non-stitched, read length $300 \mathrm{bp}$, intended output 20X, NucleomicsCore, Leuven). Read quality was tested using the software FastQC v0.11.2 [98]. Illumina PE reads that contained overrepresented sequences of adapters (TruSeq Adapter, Index 1, Illumina universal adapter [99] were adapter-trimmed with CLC assembly Cell v4.0.13 (Qiagen, Germany [100]), then quality-trimmed with CLC assembly Cell v4.0.13 (quality score minimum 20, minimum length of $\mathrm{PE}$ reads, $36 \mathrm{bp}$ ) to produce a paired-end and a single-end output [100]. Read quality was again tested using the software FastQC v0.11.2 [98].
Sequencing and read processing: PacBio A total of 10X intended sequencing output by PacBio technology was generated: $3-20 \mathrm{~kb}$ DNA from four female individuals from Specimen 9 (AFRFI4, AFRFI7, IF4, IF13) was sequenced on 8 SMRT cells using PacBio RS technology with $2 \mathrm{X}$ intended coverage (NucleomicsCore, Leuven), and $10 \mathrm{~kb}$ DNA from two other Specimen 9 females (AFRIF-19-62-III-PACB, AFRIF-63-241-IV-PACB) was sequenced on 12 SMRT cells using PacBio RSII technology with $8 \mathrm{X}$ intended coverage (BaseClear, Netherlands). The data collected from the PacBio RS instrument were processed and filtered using the SMRT Analysis software suite (PacBio, USA). The Continuous Long Read (CLR) data were filtered by Read-length $(>35)$, Subread-length (>35) and Read quality (>0.75).

Read quality was tested using the software FastQC v0.11.2 [98]. PacBio reads error correction was performed using the PE and SE trimmed inbred Illumina reads with the software LoRDEC v0.5 [101] (lordec-correct $-\mathrm{k} 21-\mathrm{s} 2$ ). Read quality was again tested using the software FastQC v0.11.2 [98].

\section{Hybrid assembly}

Previously to this publication, a non-inbred $A$. franciscana (SFB x VC) genome (SUB5823124) was assembled at the ARC as a side product of a bulked segregant analysis [36], out of paired-end (insert size 200-500 bp) and Cre-lox mate pair [102] Illumina sequencing (insert size $3 \mathrm{~kb}$, NXTGNT, Belgium, Scripps Research Institute, USA) of a mixed-sex pool of adult Artemia. Since it was constructed from Illumina sequences of a pooled sample of non-inbred diploid animals, and the genome contained many repeats (44\%), heterozygosity was high at almost $4 \mathrm{M}$ SNPs in the $1310 \mathrm{Mbp}$ genome assembly, redundancy was $41 \%$ compared to the published genome size of $0.93 \mathrm{~Gb}$ [32], and the assembly was still fragmented in 176,667 scaffolds, resulting in a fragmented annotation of 188,101 genes [36].

Therefore, the new inbred $A$. franciscana (GSL) genome described in this publication was subsequently assembled, based on Next-Generation Sequencing (NGS) data from one female and scaffolded with PacBio data from six additional females. Various assemblies were generated using different combinations of software and subsets of data. The best assembly was selected according to scaffold N50 and assembly size. The foundation of the chosen assembly was assembled using the SPAdes genome assembler v3.10.0 (read error correction 1 iteration, assembling mode, multi-cell, parameters: - $\mathrm{k}$ 21,33,55,77,99,127 ---careful) [103] with 3 input read libraries: 1) the paired-end result of the trimming (orientation: fr, interlaced, miseq or nextseq), 2) the single-end result of the trimming, 3) the single-end PacBio reads. 


\section{Scaffolding}

The SPAdes genome assembly result with K-mer size 127 was scaffolded using three different scaffolding software packages:

1) DBG2OLC v20150519 [104] was used to scaffold the genome with the inbred Artemia DNA sequencing reads: a first scaffolding round with the inbred Illumina PE and SE data, a second scaffolding round with the inbred PacBio data, and a third scaffolding round with the inbred Illumina $\mathrm{PE}$ and SE data, according to the following command lines (italic):

dbg2olc k 50 KmerCovTh 0 MinOverlap 70 LD1 0 PathCovTh 3 Contigs contigs.fasta fillumina_PE.fastq $f$ illumina_SE.fastq. dbg2olc MinLen 100 k 17 KmerCovTh 2 MinOverlap 10 AdaptiveTh 0.001 LD1 0 RemoveChimera 0 Contigs. /DBG2OLC_Consensus.fasta f pacbio.fastq. dbg2olc $k 50$ KmerCovTh 0 MinOverlap 70 LD1 0 PathCovTh 3 Contigs. /backbone_raw.fasta $f$ illumina_PE.fastq fillumina_SE.fastq.

2) L_RNA_scaffolder v20141124 [105] was used to scaffold the genome with all transcripts from the non-inbred $A$. franciscana transcriptome [34] and the following command line:

blat $/ \mathrm{dev} / \mathrm{null} / \mathrm{dev} / \mathrm{null}-\mathrm{makeOoc}=11 . \mathrm{ooc}$ -repMatch $=1024$, blat $-t=$ dna $-q=r n a-o o c=11 . o o c$ -fine -noHead, sh L_RNA_scaffolder.sh -d. -l $0.9-p 0.9$ -e 100,000 -f 1 .

3) SSPACE_Basic v2.1.1 [106] was used for rescaffolding with the inbred Illumina PE reads and the following parameters:

$-\times 0-g 3-k 3$.

\section{Gap filling and polishing}

Gaps in the scaffolded genome were filled with GapFiller v1.10 [107] using the inbred Illumina PE reads $(-\mathrm{m} 30$ -o 2 -r 0.7 -n 10 -d 50 -t 10 -g 0).
The gap-filled genome sequence was polished with 10 iterations of BWA v0.7.15 [108]. mapping and Pilon v1.21 [109] polishing to remove errors introduced by the DBG2OLC step with PacBio data (other than default parameters are: --mindepth 3 --fix bases). Pilon was run with inbred PE Illumina gDNA reads that were themselves, error-corrected using the software BrownieAligner [110]. To increase coverage at transcribed loci, an inbred RNA-seq library, dedicated to annotation was included (whole animal, MiSeq reads PE $300 \mathrm{nt}$ ). The Pilon corrections performed were limited to SNP, ambiguous nucleotide resolving, and small insertion/deletions (--fix bases).

\section{Gene annotation}

The genome was structurally annotated using several data sources, including a new inbred Artemia transcriptome assembled for that purpose.

\section{Inbred transcriptome}

Sampling $\mathrm{F}_{2}$ progeny from the inbred A. franciscana (GSL, section 1.1) was further interbred in one tank for 2 months during which cysts were continuously harvested, after which they were stored in saturated $\mathrm{NaCl}$ brine at $7{ }^{\circ} \mathrm{C}$. These cysts were transferred to a Petri dish $\left(28^{\circ} \mathrm{C}\right.$, salinity $25 \mathrm{~g} / \mathrm{L}$ Instant Ocean ${ }^{\circ}$ USA) and sampled after $0 \mathrm{~h}$ (quiescent), $4 \mathrm{~h}, 8 \mathrm{~h}, 12 \mathrm{~h}, 16 \mathrm{~h}$ and $20 \mathrm{~h}$. After $24 \mathrm{~h}$, a sample of nauplii was taken. The remaining animals were further reared (aerated seawater with added Instant Ocean ${ }^{\circ}$ USA, $28^{\circ} \mathrm{C}$, final salinity $70 \mathrm{~g} / \mathrm{L}$ using $T$. suecica as feed. After $36 \mathrm{~h}$, a sample of nauplii was taken, and the remaining animals were reared under the same conditions until sexual maturity. One adult male and one adult female were sampled. All samples were rinsed with autoclaved $\mathrm{mQ}$ on a clean sieve, put in a $1.5 \mathrm{ml}$ sterile Eppendorf tube with a sterile spatula, flash-frozen in liquid nitrogen and kept at $-80^{\circ} \mathrm{C}$.

RNA extraction For each sample, RNA was extracted by a combined method with TRIzol (Invitrogen, USA) and the RNeasy Mini Kit (Qiagen, Germany) as described previously [34], except that each freeze-dried sample was homogenized with $0.5 \mathrm{~mm}$ glass beads in $500 \mu \mathrm{l}$ of TRIzol ( $1 \mathrm{~min}$ homogenizing, $30 \mathrm{~s}$ rest, $1 \mathrm{~min}$ homogenizing), each centrifugation step was performed at $4{ }^{\circ} \mathrm{C}$, and DNA contamination was removed with the gDNA Eliminator column from the RNeasy Plus Mini Kit (Qiagen, Germany). RNA quality and yield of each sample were assessed with the Qubit ${ }^{\mathrm{nt}}$ RNA BR Assay Kit (Thermo Fisher Scientific, USA). Samples were diluted to an average of $40 \mathrm{ng} \mathrm{RNA} / \mu \mathrm{l}$. 
Sequencing and read processing All samples were pooled, and one library (500 bp) was prepared for the Illumina sequencing (Illumina Truseq stranded mRNA). The library was paired-end sequenced with Illumina MiSeq v2 500 technology (read length 250 bp, intended output 7.5 Gbp, NucleomicsCore, Leuven). Read quality was tested using the software FastQC v0.11.2 [98]. Illumina PE reads that contained overrepresented Truseq LT or HT adaptor sequences were adapter-trimmed with Trimmomatic v0.35 [111], command line:

\section{TrimmomaticPE 0.32, ILLUMINACLIP: adap- tors.fa:2:30:10 LEADING:3 TRAILING:3 MINL EN:36.}

then merged with BBMap v36.62 to produce $250 \mathrm{bp}$ long single reads [112], command line:

\section{bbmerge-auto.sh rem $k=62$ extend $2=50$ ecct vstrict.}

Transcriptome assembly Merged reads were assembled to a transcriptome with SPAdes v3.10.1 ( [113], rnaspades.py $-\mathrm{k}$ 125). A K-mer size of 125 was chosen, since the merged reads were $250 \mathrm{bp}$ long and the SPAdes developers advise using a K-mer length of half the read length for rnaSPAdes.

\section{Structural and functional genome annotation}

To support the gene model prediction, MiSeq PE $300 \mathrm{bp}$ RNAseq reads were first mapped onto the genome assembly with STAR v2.5 [114], with poor results, since no annotation file was available yet to support the mapping. Mapping was thus repeated with Hisat2 [115]. The initial mapping with Hisat2 was better, but still poor due to reads spanning multiple short exons on typical Artemia genes with long introns, the $300 \mathrm{bp}$ reads were cut back to multiple $50 \mathrm{bp}$ PE reads to avoid single reads to span more than one intron, increasing mapping rates to $65 \%$. From the resulting Hisat2 mapping with the shortened reads, intron border sequences were extracted from the genome to train SpliceMachine v1.2 using default parameters [116]. The obtained junctions (reads spanning an intron) were filtered on coverage (intron confirmed by at least 10 reads) and used as extrinsic information to support gene prediction. The software AUGUSTUS v3.3 [38] was trained (see Additional file 22: Augustus custom training files for Artemia) using the protein homology and RNA-seq provided as hints, to obtain a first draft annotation from which gene models were extracted and manually inspected to create a sufficiently large training set for EUGENE v4.2a [39]. After training, EuGene v4.2a was used with a custom parameter file for Artemia (see Additional file 23) and with the following information tracks: AUGUSTUS gene models, protein homology by BLAST+ v2.9.0 (SwissProt, Daphnia V4, Artemia proteins in NCBI, Refseq "invertebrate " [117]), transcripts from non-inbred Artemia samples [34], and RNAseq from inbred Artemia as described above. Gene models were made available online for partners, for manual curation on the ORCAE platform [118] with GenomeView [119]. Genome assembly and annotation completeness was evaluated with the software BUSCO v3, using the Eukaryota dataset [120].

\section{Identification of repeats}

To explain the difference in genome size between branchiopods, the genomes analysed for comparative genomics were analysed for genome size, repeats, transposable elements, as well as exon and intron length and small RNAs as follows. As only for a subset of the investigated organisms, repeat data sets exist and as we aimed to have homogeneous repeat data to be able to compare repeats between species, we searched and collected repeats "de novo" in each branchiopod species mentioned in Table 1 (Methods section 2), including the Artemia genome, with RepeatModeler v1.0.8(-engine ncbi) [42]. The collected repeats were further annotated/described within the RepeatModeler v1.0.8 pipeline and subsequently combined with RepBase data [121] data for completeness and to identify cross-genome repeats.

\section{Identification of mitochondrial and alien fragments}

Scaffolds of mitochondrial origin were identified by BLAST+ v2.9.0 (blastp, Bit-score $\geq 50$ ) of the $A$. franciscana mitochondrial genes [31] as indicated for homology searching [122] onto the Artemia proteome obtained by translation of the nuclear inbred genome assembly annotation. Scaffolds of equal or shorter length than the $A$. franciscana mitochondrial genome $(15,822 \mathrm{bp})$, containing only mitochondrial genes were considered "mitochondrial" to retain only nuclear genome sequences.

Scaffolds originating from alien microbial, fungal or viral organisms were eliminated from the assembly after performing BLAST + v2.9.0 (blastx) on the RefSeq nonredundant protein databases "archaea", "bacteria", "protozoa", "fungi", "viral" and "invertebrate" [123], removing only scaffolds containing $100 \%$ non-invertebrate protein with a minimum ID of $70 \%$ and a maximum length of $5 \mathrm{~kb}$ without alien hits, to avoid eliminating scaffolds containing unknown arthropod genes.

\section{Identification of functional genome content and repeats}

Functional genome content and repeats were determined as described (Methods sections 1.5.2. and 1.5.3.) based on arthropod proteome databases (see Table 1, Methods section 2). 


\section{Comparative genomics}

Based on genome contig N50 (reflective of gap occurrence and thus potential gene completeness), and taxonomic group, 11 arthropod proteomes were selected (Table 1): one chelicerate as the outgroup (Tetranychus urticae), two insects with high-quality genome assemblies, one tardigrade as the representative extremophile, and 7 crustaceans including Amphipoda, Decapoda, Copepoda and Branchiopoda, thus including all four branchiopod lineages [124]. The first aim was to characterize unique properties of Artemia as compared to three other (non-extremophile) branchiopod species. The second aim was to find similarities between extremophilic tardigrade $H$. dujardini and Artemia on the one hand, and non-extremophile crustaceans on the other hand. The third aim was to find similarities between Artemia and L. vannamei pointing to biological processes in L. vannamei for which Artemia would be a suitable model.

For the L. vannamei, H. azteca and Artemia proteomes, only the longest isoform of each protein was used respectively. All proteomes were analysed with Orthofinder v2.3.5 [125]. Gene families were constructed and potential residual transposons still present in the predicted proteome were removed from the inbred genome annotation. A Venn diagram was constructed with shared groups of orthologs (orthogroups) between Artemia and branchiopod species with the software InteractiVenn [126].

Large and small gene family expansions were analysed between Artemia and other Branchiopoda, unique genes in Artemia and Tardigrada were compared to the other species in Table 1 and non-expanded or -contracted gene families were investigated between Artemia and $L$. vannamei. Orthologous groups containing at least 5 genes in Artemia with a number of genes $\mathrm{N}$ in species $\mathrm{X}$ were considered expanded or contracted respectively, compared to species $Y$, if $N_{x} \geq 3 N_{y}$ or $3 N_{x} \leq N_{y}$. GO enrichment (Fisher's exact test, FDR $\leq 0.05$, OmicsBox) was performed onto expanded, contracted and common gene families to identify which biological processes were involved in 1) gene family expansion/contraction in Artemia compared to other Branchiopoda; 2) unique gene families in extremophiles Artemia and Tardigrada; 3) the non-expanded/contracted gene families in Artemia compared to L. vannamei. Enrichment was done with default settings: remove double ids was unchecked, rendering the Fisher's Exact Test sensitive in the direction of the test: the sequences that are present in both test-set and reference set will be deleted from the reference, but not from the test set, which can lead to zero values in the reference set. For comparative genomics with Branchiopoda, results were reduced to the most specific GO's (FDR $<0.05)$, and a bar chart with GO enrichment of up-and downregulated genes was made. For comparative genomics, with L. vannamei, a combined graph was made to show functional hierarchy of the nonexpanded/contracted gene families in Artemia compared to L. vannamei.

\section{Functional genomics: salt and anoxia tolerance-specific genes}

\section{Sampling, RNA extraction, sequencing and read processing}

All following experiments were subsequently done with A. franciscana of the strain San Francisco Bay (SFB), cyst bank ID code ARC1767. SFB was chosen and not GSL, because we were still working on the non-inbred (SFB $x$ $\mathrm{VC}$ ) genome at the time, and we had not yet sequenced the genome of the inbred Artemia (GSL).

Hydrated cysts In order to synchronize the cyst metabolic stages, the following protocol was followed: cysts were hydrated in water, dehydrated in brine, rinsed and hydrated with tap water for $1 \mathrm{~h}$ with aeration, sieved and rinsed with $\mathrm{mQ}$. Samples were taken with a small spatula cleaned with ethanol and put in labelled Eppendorfs, previously put in liquid $\mathrm{N}_{2}$ to flash-freeze. The remainder of the hydrated cysts was 1) put in filtered, nonautoclaved sea water brought to $30 \mathrm{~g} / \mathrm{L}$ in a cone with aeration in the dark at $28^{\circ} \mathrm{C}$ for $24 \mathrm{~h}$ to remove cysts hatching in the dark, the remaining cysts were left to hatch for $24 \mathrm{~h}$ under the same conditions, but in light, or 2) left to hydrate longer for a total of $6 \mathrm{~h}$ with aeration, and then used for testing under anoxic conditions.

Instar I/II nauplii, hatched in light, kept at low salinity Nauplii were separated from cysts by removing aeration for a few min, pipetting the nauplii $(25 \mathrm{ml}$ pipette) over a sieve and adding them to a new, clean, aerated cone with Instant Ocean ${ }^{\circ}$ USA $30 \mathrm{~g} / \mathrm{L}$.

Samples were taken after 15, 30, 45 and 60 min in new $30 \mathrm{~g} / \mathrm{L}$ Instant $\mathrm{Ocean}^{\circ}$ USA artificial sea water by transferring $50 \mathrm{ml}$ (with $25 \mathrm{ml}$ pipette) from a cone of nauplii over a sieve per sample time, rinsing with $\mathrm{mQ}$ over the sieve and taking $100 \mu \mathrm{l}$ of $\mathrm{mQ} /$ Artemia mix per sample with a 1-ml pipette with cut tips. Samples were flashfrozen with liquid $\mathrm{N}_{2}$. Between sampling times, the sieve was washed with tap water and rinsed with autoclaved $\mathrm{mQ}$.

To check the cysts/nauplii ratio in our samples (inevitably, some cysts or cyst shells cling to the nauplii) and the number of animals per sample, we observed $1 \mathrm{ml}$ of the $50-\mathrm{ml}$ samples and counted 93 instar I/II nauplii/ml and 9 cysts (full or empty)/ml, corresponding with 1550 nauplii per sample, each sample containing approximately $10 \%$ cysts (full or empty).

Instar I/II nauplii, hatched in light, kept at high salinity Nauplii were separated from cysts by removing 
aeration for a few min, pipetting the nauplii (25-ml pipette) over a sieve and adding them to a new, clean, aerated cone with Instant $\mathrm{Ocean}^{\circ}$ artificial brine at $200 \mathrm{~g} / \mathrm{L}$ salinity.

Samples were taken after 15, 30, 45 and $60 \mathrm{~min}$ in new $200 \mathrm{~g} / \mathrm{L}$ Instant Ocean $^{\circ}$ USA seawater by putting $50 \mathrm{ml}$ (25 ml-pipette) from a cone of nauplii over a sieve per sample time, rinsing the sieve with $\mathrm{mQ}$, and taking $100 \mu \mathrm{l}$ of $\mathrm{mQ} /$ Artemia mix per sample with a $1 \mathrm{ml}$-pipette with cut tips. Samples were flash-frozen with liquid $\mathrm{N}_{2}$. Between sampling times, the sieve was washed with tap water and rinsed with autoclaved $\mathrm{mQ}$.

Anoxic cysts Two scintillation vials with cysts hydrated for $6 \mathrm{~h}$ in $30 \mathrm{~g} / \mathrm{L}$ sea water at $28^{\circ} \mathrm{C}$ were put under anoxic conditions in the light on a rotor. Anoxic conditions were reached according to a modified protocol [16]. In scintillation glass vials of $8 \mathrm{ml}, 70-80 \mathrm{mg}$ dry cysts were placed. $\mathrm{N}_{2}$ gas was flushed through the vial to remove the oxygen in the vial, then the vial was capped and set aside, allowing the $\mathrm{N}_{2}$ to seep into the porous parts of the outer cyst shell. $\mathrm{N}_{2}$ was bubbled through buffer $(0.25 \mathrm{M} \mathrm{NaCl}$ in $0.05 \mathrm{M}$ phosphate buffer, $\mathrm{pH} 7.2)$ during a minimum of $4 \mathrm{~h}$. With the $\mathrm{N}_{2}$ still bubbling, 6 $\mathrm{ml}$ of the anoxic solution was transferred into each vial without any air leaking into the vial and Parafilm was wrapped around the tightly shut vial cap. Vials were oscillated on a rotating platform shaker (50 revolutions/ $\mathrm{min}$ ) for the first $24 \mathrm{~h}$ to hydrate the cysts completely and to ascertain that even traces of oxygen (if present) were consumed. The vials containing the anoxic, hydrated cysts were stored at room temperature and in ambient light.

Samples were taken after $25 \mathrm{~h}$ of anoxia ( 1 supplementary hour after metabolic activity stops). The content of each vial was sieved and rinsed with $\mathrm{mQ}$. Per sample, $100 \mu \mathrm{l}$ of $\mathrm{mQ} /$ Artemia mix was taken with a 1-ml pipette with cut tips. Samples were flash-frozen with liquid $\mathrm{N}_{2}$. Between sampling times, the sieve was washed with tap water and rinsed with autoclaved $\mathrm{mQ}$.

The anoxic state in the scintillation vials was verified indirectly by observing the cysts under a binocular microscope after 3 days of anoxia. In a sample of 500 cysts, there was one clear umbrella and a few $[2,3]$ cysts possibly in the breaking stage. In similar conditions, but in the presence of oxygen, the same sample had a hatching percentage of $70 \%$ after $24 \mathrm{~h}$. Crushing the cysts between microscope slides to push the non-hatched embryos out of their cyst shells was done to verify that practically all cysts contained an embryo and that the results were not biased by an excessive amount of empty cyst shells.

A. franciscana from the San Francisco Bay strain (SFB) were sampled under four different conditions, including different life cycle stages, and analysed (Table 2). RNA extraction of each sample, sequencing and read processing have previously been described [34]. Briefly, RNA was extracted from each freeze-dried, homogenized sample by a combined method with TRIzol (Invitrogen, USA), the RNeasy Mini Kit (Qiagen, Germany) and the DNase I Kit (Qiagen, Germany) [127]. RNA quality and yield of each sample were assessed with the Agilent 2100 bioanalyzer (Agilent Technologies, USA). Samples were diluted to an average of $40 \mathrm{ng}$ RNA/ $\mu$ l. The RNA sample libraries were prepared by subsequent use of the TruSeq $^{\text {Tim }}$ mRNA enrichment and TruSeq ${ }^{\mathrm{mm}}$ RNA sample preparation kits (Illumina, USA) and paired-end sequenced at the Genomics Core facility (UZ Leuven, http://gc.uzleuven.be) on an Illumina HiSeq 2000 instrument $(2 \times 100 \mathrm{bp}$, unstranded, insert size 200). Reads of each RNAseq library were quality-trimmed with CLC Assembly Cell v4.06 software [100].

\section{Mapping and summarization}

FastQC v 0.11.2 and MultiqQC, v1.7 [128] were used to evaluate the sequencing data quality $[98,128]$. Two distinct GC content profiles were observed (Additional file 24: Sequence GC-content profiles for all samples used for differential expression analysis). Although both profiles are not problematic on their own, they clearly indicate a batch effect, possibly due to a separate library prep by the sequencing provider. As the batch effect was not associated with the contrasts of interest, yet constituted an unwanted source of variance, we took it into consideration during differential expression analysis as a random factor. Otherwise, no consistent infringements on data quality were identified. STAR v2.6.1c) was used to perform sequence alignment. To maximally preserve information, multi mapped reads were included in read summarization with the software featureCounts version1.5.2 [129].

\section{Differential expression}

The "high salinity" and "anoxia" conditions (Table 2) were examined for differential expression.

Samples were annotated based on life cycle stage, time point at which the sample was taken, salinity level of the treatment, light environment of the treatment and the batch effect, a technical variation source introduced by necessity in high-throughput studies (Table 5). Samples where a certain property was not tested are indicated as NA (non-applicable).

High salinity and anoxia models For high salinity, after filtering of non-informative genes, i.e., counts per million equivalent $<3$ for over 14 out of 16 samples, the following model was fitted to the expression data (with the 
Table 5 Annotation of A. franciscana samples for differential expression analysis under different salinities and oxygen levels

\begin{tabular}{|c|c|c|c|c|c|}
\hline Sample & Life cycle state & Time point (min) & Salinity & Light & Batch \\
\hline HSR000198 & cysts (anoxia) & 60 & NA & NA & 1 \\
\hline HSR000210 & cysts (anoxia) & 60 & NA & NA & 1 \\
\hline HSR000222 & cysts (anoxia) & 60 & NA & NA & 1 \\
\hline HSR000190 & nauplii & 15 & low & light & 2 \\
\hline HSR000202 & nauplii & 15 & low & light & 1 \\
\hline HSR000214 & nauplii & 15 & low & light & 2 \\
\hline HSR000191 & nauplii & 30 & low & light & 2 \\
\hline HSR000203 & nauplii & 30 & low & light & 1 \\
\hline HSR000215 & nauplii & 30 & low & light & 2 \\
\hline HSR000192 & nauplii & 45 & low & light & 2 \\
\hline HSR000204 & nauplii & 45 & low & light & 1 \\
\hline HSR000216 & nauplii & 45 & low & light & 2 \\
\hline HSR000193 & nauplii & 60 & low & light & 2 \\
\hline HSR000205 & nauplii & 60 & low & light & 1 \\
\hline HSR000217 & nauplii & 60 & low & light & 2 \\
\hline HSR000194 & nauplii & 15 & high & light & 2 \\
\hline HSR000206 & nauplii & 15 & high & light & 1 \\
\hline HSR000218 & nauplii & 15 & high & light & 2 \\
\hline HSR000195 & nauplii & 30 & high & light & 2 \\
\hline HSR000207 & nauplii & 30 & high & light & 1 \\
\hline HSR000219 & nauplii & 30 & high & light & 2 \\
\hline HSR000196 & nauplii & 45 & high & light & 2 \\
\hline HSR000208 & nauplii & 45 & high & light & 1 \\
\hline HSR000220 & nauplii & 45 & high & light & 2 \\
\hline HSR000197 & nauplii & 60 & high & light & 2 \\
\hline HSR000209 & nauplii & 60 & high & light & 1 \\
\hline HSR000221 & nauplii & 60 & high & light & 2 \\
\hline
\end{tabular}

first batch, a low salinity treatment and the time point of $15 \mathrm{~min}$ as a reference):

$$
\begin{aligned}
& Y=\beta_{0}+\beta_{B} X_{B}+\beta_{S} X_{S}+\beta_{T 30} X_{T 30}+\beta_{T 45} X_{T 45} \\
& \quad+\beta_{T 60} X_{T 60} \\
& +\beta_{S: T 30} X_{S} X_{T 30}+\beta_{S: T 45} X_{S} X_{T 45}+\beta_{S: T 60} X_{S} X_{T 60}
\end{aligned}
$$

With $\beta_{\mathrm{B}}$ : batch effect of second batch.

$\beta_{\mathrm{S}}$ : high salinity treatment effect.

$\beta_{\mathrm{T} 30}$ : time effect after $30 \mathrm{~min}$ (compared to $15 \mathrm{~min}$ ).

$\beta_{\mathrm{T} 45}$ : time effect after $45 \mathrm{~min}$ (compared to $15 \mathrm{~min}$ ).

$\beta_{\mathrm{T} 60}$ : time effect after $60 \mathrm{~min}$ (compared to $15 \mathrm{~min}$ ).

This way, the effect of the salinity could be assessed at each time point separately. As the design is rather complex, a stage-wise testing approach as described by Van Den Berghe et al. was performed, thus boosting power for the interaction effects [130]. Briefly, an omnibus test was first performed to assess for which genes at least one of the contrasts of interest shows a significant difference (analogously to ANOVA). The contrasts of interest were the effect of salinity on each time point separately, and the average effect over all time points. Subsequently, a post-hoc analysis was performed for each contrast separately with an adjusted $p$-value.

For anoxia, after filtering of non-informative genes, i.e., counts per million $<3$ for over 3 out of 6 samples, a simple model only considering the effect of anoxia compared to hydrated cysts was used. No batch effect was present in samples studied under anoxia.

Normalization was performed using trimmed mean of M-values (TMM), and FDR adjustment by Benjamini-Hochberg's method, as default for edgeR v3.24.3, which was used for both omnibus test and post-hoc testing [131]. Genes with padjScreen $<0.05$ (salinity) or PVal $<0.05$ (anoxia) were considered differentially expressed and used for subsequent enrichment analyses. 


\section{Enrichment analysis}

For each contrast, GO names (biological process, molecular process and cellular component), were analysed for enrichment $(F D R \leq 0.05)$ with Fisher's exact test in OmicsBox [132] using the ID list of the selected DEG as the test set and the Artemia proteome (imported to OmicsBox, default InterProScan annotated, annotations merged) as the reference set. Enrichment was done with default settings: remove double ids was unchecked, rendering the Fisher's Exact Test sensitive in the direction of the test: the sequences that are present in both testset and reference set will be deleted from the reference, but not from the test set, which can lead to zero values in the reference set. Results were reduced to the most specific GO's (FDR $\leq 0.05)$, and a bar chart with GO enrichment of differentially expressed genes was made. Pathway enrichment was performed with the online GUI software STRING v11.0 using default settings and model organism Daphnia pulex [133] and using "multiple sequences" as input: DEG under high salinity and a subset of DEG under anoxia containing only genes with a functional annotation (to conform to the $2 \mathrm{~K}$ sequence maximum of the platform) [133]. STRING first produced a "STRING annotation" by matching each protein set with $D$. pulex proteins in its database, only the best match was kept. Subsequently, STRING performed pathway enrichment with a stringent cut-off $(F D R \leq 0.05)$ on each $D$. pulex gene. Interesting enriched pathways were drawn with the online GUI software KEGG Mapper Search Pathway function [134] in the organism-specific mode (dpx).

\section{Supplementary Information}

The online version contains supplementary material available at https://doi. org/10.1186/s12864-021-07937-z.

Additional file 1. Assembly characteristics of all assembled crustacean genomes. Characteristics listed are: species, whether the species genome is annotated yes or no, N50 of the fragments with the highest assembly hierarchy, number of fragments with the highest assembly hierarchy in the assembly, haploid genome size, assembly size, completeness of the assembly (=haploid GS/assembly size), taxonomic lineage (NCBI taxonomy), reference for the genome paper.

Additional file 2. Evolution of Artemia assembly quality metrics throughout the assembly steps. Evolution of the scaffold N50, the number of fragments and the genome completeness (assembly size/ genome size) in the subsequent Artemia assembly stages

Additional file $\mathbf{3}$ BUSCO analysis results for the A. franciscana genome assembly and annotation.

Additional file 4. BLAST results for mitochondrial genes in the Artemia genome. Listed: Query accession and gene name, presence of a (significant) BLAST hit in the Artemia proteome with the highest bit score, E-value and bit score of the hit, scaffold length of the scaffold on which the hit lies, percentage of mitochondrial genes on this scaffold.

Additional file 5. Taxonomic groups of alien genomes identified in the Artemia genome.

Additional file $\mathbf{6}$ Expanded or contracted Artemia orthogroups compared to other Branchiopoda. Listed: Orthogroup ID, number of genes in this orthogroup in A. franciscana, D. pulex, L. arcticus, and $E$. texana, expanded or contracted status of the orthogroup in Artemia compared to D. pulex, L. arcticus and E. texana, conservation in Branchiopoda (whether this orthogroup contains genes for each branchiopod), comma-separated IPR description of Artemia genes in this orthogroup, Artemia genes in this orthogroup.

Additional file $\mathbf{7} \mathrm{GO}$ enrichment of Artemia compared to other Branchiopoda. Listed: GO ID, name and category, false discovery rate (FDR) and $P$ value of the Fisher's exact test enrichment analysis in Blast2GO, number of Artemia genes from expanded/contracted orthogroups in this GO ID, number of whole Artemia genome genes in this GO category, number of Artemia genes from expanded/contracted orthogroups in this GO ID without GO annotation. The Fisher's Exact Test is sensitive in the direction of the test: the genes that are present in the test-set and also in the reference genome set will be deleted from the reference, but not from the test set, resulting in zero sequences in the reference set and values above zero in the test set. Significantly enriched GOs (FDR $\leq 0.05$, biological process) of Artemia genes in expanded or contracted orthogroups compared to Branchiopoda are given.

Additional file 8 Expanded or contracted Artemia and $\mathrm{H}$. dujardini orthogroups compared to other Arthropoda. Listed: Orthogroup ID, number of genes in this orthogroup in A. franciscana and in the other arthropod species, expanded or contracted status of the orthogroup in Artemia compared to the other arthropod species, comma-separated IPR description of Artemia genes in this orthogroup, $\mathrm{H}$. dujardini genes in this orthogroup, Artemia genes in this orthogroup.

Additional file 9. STAR mapping statistics for differential expression analysis in Artemia. Listed: sample name, total number of reads for this sample, percentage of uniquely mapped reads, absolute number of uniquely mapped reads, percentage of multi mapped reads, absolute number of multi mapped reads.

Additional file 10. Summarization statistics for differential expression analysis in Artemia. Listed: sample name, total counts, percentage of counts assigned to a gene annotation, absolute counts assigned to a gene annotation. ${ }^{*}$ notice that this amount can be more than the sum of uniquely mapped + multi-mapped in the mapping statistics since multimapped reads are considered.

Additional file 11 Differentially expressed genes under high salinity $(p<0.05)$. Listed: functional annotation of the differentially expressed gene, gene ID in the genome annotation and on the ORCAE platform, $p$ value, average log fold change of gene expression under high salinity, gene regulation of the differentially expressed gene (up or down), InterPro description of the gene family to which the gene belongs.

Additional file 12 Differentially expressed genes under anoxia $(p<0.05)$ Listed: functional annotation of the differentially expressed gene, gene ID in the genome annotation and on the ORCAE platform, $p$ value, log fold change of gene expression under anoxia, gene regulation of the differentially expressed gene (up or down), InterPro description of the gene family to which the gene belongs.

Additional file $\mathbf{1 3} \mathrm{GO}$ enrichment in Artemia under high salinity. Significantly Enriched GOs (FDR $\leq 0.05$ ) of Artemia genes differentially expressed under high salinity. Listed: GO ID, name and category, false discovery rate (FDR) and $P$ value of the Fisher's exact test enrichment analysis in Blast2GO, number of DEG under high salinity in this GO category, number of whole Artemia genome genes in this GO category, number of DEG under high salinity without GO annotation. The Fisher's Exact Test is sensitive in the direction of the test: the genes that are present in the test-set and also in the reference genome set will be deleted from the reference, but not from the test set, resulting in zero sequences in the reference set and values above zero in the test set.

Additional file $\mathbf{1 4}$ Pathway enrichment in Artemia under high salinity. Significantly enriched (Fisher's exact test corrected for multiple testing, FDR $\leq 0.05$ ) pathways of Artemia genes differentially expressed under high salinity. Listed in first worksheet (STRING annotation): gene number, ORCAE gene ID, STRING Daphnia pulex gene ID, BLAST identity and bit score, gene name and gene annotation. Listed in second worksheet (STRING pathway enrichment): KEGG Daphnia pulex pathway name, pathway description, number of DEG under high salinity in this pathway, 
number of genes in the $D$. pulex genome that belong to this pathway, enrichment FDR, matching $D$. pulex gene IDs, matching gene names in pathways shown in figures and additional files, matching $D$. pulex gene ID labels.

Additional file 15. Consolidation of DEG analysis, GO enrichment and pathway enrichment in Artemia under high salinity.

Additional file 16. The enriched Carbon metabolism pathway in Artemia under high salinity. Up- and downregulated genes are indicated on the KEGG map dpx01200.

Additional file 17. $\mathrm{GO}$ enrichment in Artemia under anoxia. Significantly enriched GOs (FDR $\leq 0.05$ ) of Artemia genes differentially expressed under anoxia. Listed: GO ID, name and category, false discovery rate (FDR) and $P$ value of the Fisher's exact test enrichment analysis in Blast2GO, number of DEG under anoxia in this GO ID, number of whole Artemia genome genes in this $G O I D$, number of DEG under anoxia without $G O$ annotation. The Fisher's Exact Test is sensitive in the direction of the test: the genes that are present in the test set and also in the reference genome set will be deleted from the reference, but not from the test set, resulting in zero sequences in the reference set and values above zero in the test set.

Additional file 18 Pathway enrichment in Artemia under anoxia. Significantly enriched (Fisher's exact test corrected for multiple testing, FDR $\leq 0.05$ ) pathways of Artemia genes differentially expressed under anoxia. Listed in first worksheet (STRING annotation): gene number ORCAE gene ID, STRING Daphnia pulex gene ID, BLAST identity and bit score, gene name and gene annotation. Listed in second worksheet (STRING pathway enrichment): KEGG Daphnia pulex pathway name, pathway description, number of DEG under anoxia in this pathway, number of genes in the $D$. pulex genome that belong to this pathway, enrichment FDR, matching $D$. pulex gene IDs, matching gene names in pathways shown in figures and additional files, matching $D$. pulex gene ID labels.

Additional file 19. Consolidation of DEG analysis, $\mathrm{GO}$ enrichment and pathway enrichment in Artemia under anoxia.

Additional file 20. The enriched N-glycan biosynthesis pathway in Artemia under anoxia. Up- and downregulated genes are indicated on the KEGG map dpx00510.

Additional file 21. The enriched Basal transcription factors pathway in Artemia under anoxia. Up- and downregulated genes are indicated on the KEGG map dpx03022.

Additional file 22. Augustus custom training files for Artemia. Includes probabilities, parameters and weights used for Augustus training for annotation of the Artemia genome.

Additional file 23. EuGene custom parameter file for Artemia. Includes parameters used for EuGene training for annotation of the Artemia genome.

Additional file $\mathbf{2 4}$. Sequence GC-content profiles for all samples used for differential expression analysis.

\section{Acknowledgements}

Anita De Haese (survival experiments and sampling for RNA), Christ Mahieu (inbred Artemia rearing support), Ilse Vercauteren (RNA extractions), HPCUGent, supported by the Flemish Supercomputer Center (Computer cluster), Cedric Trappeniers (KEGG Pathway mapping).

\section{Dedication}

We would like to dedicate this paper to the memory of a colleague and friend Dr. Tom MacRae, who passed away during the course of 2020 .

\section{Authors' contributions}

SDV performed study design for genome sequencing and assembly, performed and interpreted data analyses (sequencing, read processing, genome assembly, transcriptome assembly, mitochondrial fragment identification, GO and pathway enrichment for differential expression and comparative genomics), interpreted data analyses (genome characterization, genome annotation, alien fragment identification, differential expression comparative genomics), wrote the paper, except part of the methods (genome polishing and annotation, repeat identification, differential expression) and reviewed the paper. SR supported study design for genome sequencing and assembly, performed data analyses (genome polishing, structural and functional genome annotation, repeat identification, alien fragment identification), wrote part of the methods (genome polishing, genome annotation) and reviewed parts of the paper. LC performed the statistics for differential expression analysis, wrote part of the methods (differential expression) and reviewed parts of the paper. WD performed data selection and the InterProScan run for the comparative genomics section and reviewed parts of the paper. MV supported study design for genome sequencing and reviewed the paper. PS supported study design for genome sequencing and reviewed the paper. JSC provided the appropriate anoxia treatment protocol. ZN provided the inbred Artemia strain and reviewed the paper. FVN provided the cre-lox mate-pair sequencing technology. PN performed and optimized the DNA extraction protocol to obtain long DNA strings for sequencing with PacBio long read technology and reviewed the paper. GVS supported study design for genome sequencing and reviewed the paper. YVDP supported study design for genome sequencing and functional genomics and reviewed the paper. TVL supported the comparative genomics study of the genome. TDM supported the differential expression study of the genome and reviewed parts of the paper. PB supported study design for genome sequencing and functional genomics and extensively reviewed the paper. All authors read and approved the final manuscript.

\section{Funding}

The design of the study was funded by the Flemish government Special Research Fund (Bijzonder Onderzoeksfonds, BOF, project number 01 J08209) and collection, analysis, and interpretation of data and writing of the manuscript were funded by the Laboratory of Aquaculture \& Artemia Reference Center.

\section{Availability of data and materials}

The datasets supporting the conclusions of this article are available in the GENBANK repository, Inbred A. franciscana genome:

SUB6538872.

Bioproject PRJNA589114.

Inbred A. franciscana transcriptome:

Bioproject PRJNA589261.

This submission is deposited in Genbank under the code

"JAGUUB000000000" and will be made public after acceptation of this paper. For reviewers of this paper, a confidential account was made on our genome platform Orcae: https://bioinformatics.psb.ugent.be/orcae/ login: artemia.

password: AuBSvP.

The sequencing read datasets used and/or analysed during the current study are available from the corresponding author on reasonable request.

\section{Declarations}

\section{Ethics approval and consent to participate}

This study complied with the guidelines Directive 2010/63/EU in Europe for the involvement of animals in the study.

\section{Consent for publication}

Not applicable.

Competing interests

The authors declare that they have no competing interests.

\section{Author details}

${ }^{1}$ Laboratory of Aquaculture \& Artemia Reference Center, Department of Animal Sciences and Aquatic Ecology, Faculty of Bioscience Engineering, Ghent University, Ghent, Belgium. ²Department of Plant Systems Biology, VIB, Department of Biotechnology and Bioinformatics, Ghent University, Ghent, Belgium. ${ }^{3}$ Department of Data Analysis and Mathematical Modelling, Faculty of Bioscience Engineering, Ghent University, Ghent, Belgium. ${ }^{4}$ Department of Plants and Crops, Faculty of Bioscience Engineering, Ghent University, Ghent, Belgium. ${ }^{5} \mathrm{GNOMIXX}$, Boterbloemstraat 26, 9090 Melle, Belgium. ${ }^{6}$ Coastal and Marine Sciences Institute, University of California, Bodega Bay, Davis, CA, USA ${ }^{7}$ Department of Medical Technology, School of Health Sciences, University of Occupational and Environmental Health, Japan, Kitakyushu, Fukuoka, Japan. 
${ }^{8}$ Department of Pharmaceutics, Faculty of Pharmaceutical Sciences, Ghent University, Ghent, Belgium. 'Laboratory for Immunology and Animal Biotechnology, Department of Animal Sciences and Aquatic Ecology, Faculty of Bioscience Engineering, Ghent University, Ghent, Belgium. ${ }^{10} \mathrm{Centre}$ for Microbial Ecology and Genomics, Department of Biochemistry, Genetics and Microbiology, University of Pretoria, Pretoria, South Africa.

\section{Received: 22 January 2021 Accepted: 14 August 2021}

\section{Published online: 31 August 2021}

\section{References}

1. Lavens P, Sorgeloos P. Manual on the production and use of live food for aquaculture. In: FAO, editor. FAO Fisheries Technical Paper 361. Rome: FAO; 1996. p. 295.

2. Kayim M, Ates $M$, Elekon $H A$. The effects of different feeds under the same salinity conditions on the growth and survival rate of Artemia. J Anim Vet Adv. 2010:9(8):1223-6.

3. Leger $P$, Bengtson DA, Simpson $K L$, Sorgeloos $P$. The use and nutritional value of Artemia as a food source. Oceanogr Mar Biol. 1986;24:521-623.

4. Asem A, Rastegar-Pouyani N, De Los Ríos-Escalante P. The genus Artemia leach, 1819 (Crustacea: Branchiopoda). I. True and false taxonomical descriptions. Lat Am J Aquat Res. 2010;38(3):501-6.

5. Maniatsi S, Baxevanis AD, Kappas I, Deligiannidis P, Triantafyllidis A, Papakostas $\mathrm{S}$, et al. Is polyploidy a persevering accident or an adaptive evolutionary pattern? The case of the brine shrimp Artemia. Mol Phylogenet Evol. 2011;58(2):353-64. https://doi.org/10.1016/.jympev.2010.11.029.

6. Ollivier B, Caumette P, Garcia JL, Mah RA. Anaerobic bacteria from hypersaline environments. Microbiol Rev. 1994;58(1):27-38. https://doi.org/1 0.1128/mr.58.1.27-38.1994.

7. Jain CK. Salinity. In: Singh VP, Singh P, Haritashya UK, editors. Encyclopedia of Snow, Ice and Glaciers. Dordrecht: Springer Netherlands; 2011. p. 959.

8. Plemenitas A, Lenassi M, Konte T, Kejzar A, Zajc J, Gostincar C, et al. Adaptation to high salt concentrations in halotolerant/halophilic fungi: a molecular perspective. Front Microbiol. 2014;5:199.

9. Harding T, Roger AJ, Simpson AGB. Adaptations to high salt in a halophilic protist: differential expression and gene acquisitions through duplications and gene transfers. Front Microbiol. 2017:8:944. https://doi.org/10.3389/ fmicb.2017.00944.

10. DasSarma S, DasSarma P. Halophiles and their enzymes: negativity put to good use. Curr Opin Microbiol. 2016;25:120-6.

11. Gharat SA, Parmar S, Tambat S, Vasudevan M, Shaw BP. Transcriptome analysis of the response to $\mathrm{NaCl}$ in Suaeda maritima provides an insight into salt tolerance mechanisms in halophytes. PLoS One. 2016;1 1(9): e0163485. https://doi.org/10.1371/journal.pone.0163485.

12. Gajardo GM, Beardmore JA. The brine shrimp artemia: adapted to critical life conditions. Front Physiol. 2012;3:185.

13. Mitchell B, Crews ST. Expression of the Artemia trachealess gene in the salt gland and epipod. Evol Dev. 2002;4(5):344-53. https://doi.org/10.1046/j.152 5-142X.2002.02023.X.

14. Mahfouz ME, El-Gamal MM, Shraby SM. Morphology and anatomical structure of the larval salt gland of Artemia tunisiana under different salinities. Afr J Biotechnol. 2013;12(42):6032-41.

15. Schrehardt A. A scanning electron-microscope study of the post-embryonic development of Artemia. In: Sorgeloos P, Bengtson DA, Decleir W, Jaspers E, editors. Artemia Research and its Applications Morphology, Genetics, Strain Characterization, Toxicology. 1. Wetteren: Universa Press; 1987. p. 5-32.

16. Clegg JS. Embryos of Artemia franciscana survive four years of continuous anoxia: the case for complete metabolic rate depression. J Exp Biol. 1997; 200(3):467-75. https://doi.org/10.1242/jeb.200.3.467.

17. Spooner BS, DeBell L, Armbrust L, Guikema JA, Metcalf J, Paulsen A. Embryogenesis, hatching and larval development of Artemia during orbital spaceflight. Adv Space Res. 1994;14(8):229-38. https:/doi.org/10.1016/0273-1177(94)90407-3.

18. Browne RA, Bowen ST. Taxonomy and population genetics of Artemia. In: Artemia biology. Boca Raton: CRC Press; 1991. p. 221-35.

19. Robbins HM, Van Stappen G, Sorgeloos P, Sung YY, MacRae TH, Bossier P. Diapause termination and development of encysted Artemia embryos: roles for nitric oxide and hydrogen peroxide. J Exp Biol. 2010;213(9):1464-70. https://doi.org/10.1242/jeb.041772.

20. Clegg JS. Desiccation tolerance in encysted embryos of the animal extremophile, Artemia. Integr Comp Biol. 2005;45(5):715-24. https://doi.org/1 0.1093/icb/45.5.715.
21. Hengherr S, Schill RO, Clegg JS. Mechanisms associated with cellular desiccation tolerance in the animal extremophile Artemia. Physiol Biochem Zool. 2011;84(3):249-57. https://doi.org/10.1086/659314.

22. Toxopeus J, Warner AH, MacRae TH. Group 1 LEA proteins contribute to the desiccation and freeze tolerance of Artemia franciscana embryos during diapause. Cell Stress Chaperones. 2014;19(6):939-48. https://doi.org/10.1007/ s12192-014-0518-3.

23. LeBlanc BM, Le MT, Janis B, Menze MA, Hand SC. Structural properties and cellular expression of AfrLEA6, a group 6 late embryogenesis abundant protein from embryos of Artemia franciscana. Cell Stress Chaperones. 2019; 24(5):979-90. https://doi.org/10.1007/s12192-019-01025-8.

24. Ono F, Mori Y, Takarabe K, Fujii A, Saigusa M, Matsushima Y, et al. Effect of ultra-high pressure on small animals, tardigrades and Artemia. Cogent Physics. 2016;3(1):1167575.

25. Clegg JS. Protein stability in Artemia embryos during prolonged anoxia. Biol Bull. 2007;212(1):74-81. https://doi.org/10.2307/25066582.

26. Chavez M, Landry C, Loret S, Muller M, Figueroa J, Peers B, et al. APH-1, a POU homeobox gene expressed in the salt gland of the crustacean Artemia franciscana. Mech Dev. 1999;87(1-2):207-12. https://doi.org/10.1016/50925-4 773(99)00152-5.

27. Dai JQ, Zhu XJ, Liu FQ, Xiang JH, Nagasawa H, Yang WJ. Involvement of p90 ribosomal 56 kinase in termination of cell cycle arrest during development of Artemia-encysted embryos. J Biol Chem. 2008;283(3):1705-12. https://doi. org/10.1074/jbc.M707853200.

28. Li S, Chakraborty N, Borcar A, Menze MA, Toner M, Hand SC. Late embryogenesis abundant proteins protect human hepatoma cells during acute desiccation. Proc Natl Acad Sci U S A. 2012;109(51):20859-64. https:// doi.org/10.1073/pnas.1214893109.

29. NCBI taxonomy [June 2013]. Available from: http://www.ncbi.nlm.nih.gov/Ta xonomy/Browser. Accessed June 2013.

30. Stillman JH, Colbourne JK, Lee CE, Patel NH, Phillips MR, Towle DW, et al. Recent advances in crustacean genomics. Integr Comp Biol. 2008;48(6):85268. https://doi.org/10.1093/icb/icn096.

31. Valverde JR, Batuecas B, Moratilla C, Marco R, Garesse R. The complete mitochondrial-DNA sequence of the crustacean Artemia-Franciscana. J Mol Evol. 1994;39(4):400-8. https://doi.org/10.1007/BF00160272.

32. De Vos S, Bossier P, Van Stappen G, Vercauteren I, Sorgeloos P, Vuylsteke M. A first AFLP-based genetic linkage map for brine shrimp Artemia franciscana and its application in mapping the sex locus. PLoS One. 2013;8(3):e57585. https://doi.org/10.1371/journal.pone.0057585.

33. Zhang Y, Wang D, Zhang Z, Wang Z, Zhang D, Yin H. Transcriptome analysis of Artemia sinica in response to Micrococcus lysodeikticus infection. Fish Shellfish Immunol. 2018;81:92-8. https://doi.org/10.1016/j.fsi.2018.06. 033.

34. De Vos S, Van Stappen G, Sorgeloos P, Vuylsteke M, Rombauts S, Bossier P. Identification of salt stress response genes using the Artemia transcriptome. Aquaculture. 2019:500:305-14. https:/doi.org/10.1016/..aquaculture.2018.09.067.

35. Colbourne JK, Pfrender ME, Gilbert D, Thomas WK, Tucker A, Oakley TH, et al. The ecoresponsive genome of Daphnia pulex. Science. 2011;331(6017): 555-61. https://doi.org/10.1126/science.1197761.

36. De Vos S. Genomic tools and sex determination in the extremophile brine shrimp Artemia franciscana. Ghent: Ghent University; 2014.

37. Cruces J, Wonenburger MLG, Díaz-Guerra M, Sebastián J, Renart J. Satellite DNA in the crustacean Artemia. Gene. 1986;44(2-3):341-5. https://doi.org/1 0.1016/0378-1119(86)90200-3.

38. Hoff KJ, Stanke M. Predicting genes in single genomes with AUGUSTUS. Curr Protoc Bioinformatics. 2019;65(1):e57. https://doi.org/10.1002/cpbi.57.

39. Schiex T, Moisan A, Rouzé P. EuGene: An Eucaryotic Gene Finder that combines several sources of evidence. In: Gascuel O, Sagot L, editors. Computational Biology; 2001. p. 111-25.

40. Veeckman E, Ruttink T, Vandepoele K. Are we there yet? Reliably estimating the completeness of plant genome sequences. Plant Cell. 2016;28(8):175968. https://doi.org/10.1105/tpc.16.00349.

41. Rhoads A, Au KF. PacBio sequencing and its applications. Genomics Proteomics Bioinformatics. 2015;13(5):278-89. https://doi.org/10.1016/.j.gpb.2015.08.002.

42. Smit A, Hubley R. RepeatModeler Open-1.0. (2008-2015); 2018.

43. Tarailo-Graovac M, Chen N. Using RepeatMasker to identify repetitive elements in genomic sequences. Curr Protoc Bioinform. 2009;4:4-10.

44. Ye Z, Xu S, Spitze K, Asselman J, Jiang X, Ackerman MS, et al. A New Reference Genome Assembly for the Microcrustacean Daphnia pulex. G3. 2017;7(5):1405-16. 
45. Savojardo C, Luchetti A, Martelli PL, Casadio R, Mantovani B. Draft genomes and genomic divergence of two Lepidurus tadpole shrimp species (Crustacea, Branchiopoda, Notostraca). Mol Ecol Resour. 2019;19(1):235-44. https://doi.org/10.1111/1755-0998.12952.

46. Baldwin-Brown JG, Weeks SC, Long AD. A new standard for crustacean genomes: the highly contiguous, annotated genome assembly of the clam shrimp Eulimnadia texana reveals HOX gene order and identifies the sex chromosome. Genome Biol Evol. 2018;10(1):143-56. https://doi.org/10.1093/ gbe/evx280.

47. The genome of the Antarctic-endemic copepod, Tigriopus kingsejongensis. 2017 [cited 2019]. Available from: http://gigadb.org/dataset/100249. Accessed 2019.

48. Index of /genomes/Penaeus_vannamei/protein/. Institute of Oceanology CAoS. 2018 [cited 2019]. Available from: ftp://ftp.ncbi.nlm.nih.gov/genomes/ Penaeus_vannamei/. Accessed 2019.

49. Medicine BCo: Index of /genomes/Hyalella_azteca/protein/. 2016 [cited 2019]. Available from: ftp://ftp.ncbi.nih.gov/genomes/Hyalella_azteca/ protein/. Accessed 2019.

50. Yoshida Y, Koutsovoulos G, Laetsch DR, Stevens L, Kumar S, Horikawa DD, et al. Comparative genomics of the tardigrades Hypsibius dujardini and Ramazzottius varieornatus. PLoS Biol. 2017;15(7):e2002266. https://doi.org/1 0.1371/journal.pbio.2002266.

51. Proteomes - Drosophila melanogaster (Fruit fly). C. D S. 2019 [cited 2019]. Available from: https://www.uniprot.org/proteomes/UP000000803:. Accessed 2019.

52. Proteomes - Bombyx mori (Silk moth). Consortium TISG. 2018 [cited 2019]. Available from: https://www.uniprot.org/proteomes/UP000005204. Accessed 2019.

53. Proteomes - Tetranychus urticae (Two-spotted spider mite). S. R. 2011 [cited 2019]. Available from: https://www.uniprot.org/proteomes/UP000015104. Accessed 2019.

54. Li Z, Xu X. Post-translational modifications of the mini-chromosome maintenance proteins in DNA Replication. Genes. 2019;10(5):331.

55. $\mathrm{Wu} \mathrm{H}, \mathrm{Li}$ Z. The importance of $\mathrm{Cl}(-)$ exclusion and vacuolar $\mathrm{Cl}(-)$ sequestration: revisiting the role of $\mathrm{cl}(-)$ transport in plant salt tolerance. Front Plant Sci. 2019;10:1418. https://doi.org/10.3389/fpls.2019.01418.

56. Evans TG, Kultz D. The cellular stress response in fish exposed to salinity fluctuations. J Exp Zool A Ecol Integr Physiol. 2020;333(6):421-35. https://doi. org/10.1002/jez.2350.

57. Baral A, Shruthi KS, Mathew MK. Vesicular trafficking and salinity responses in plants. IUBMB Life. 2015;67(9):677-86. https://doi.org/10.1002/iub.1425.

58. Robinson DG, Pimpl P. Clathrin and post-Golgi trafficking: a very complicated issue. Trends Plant Sci. 2014;19(3):134-9. https://doi.org/10.101 6/j.tplants.2013.10.008

59. Tollefsen KE, Song Y, Kleiven M, Mahrosh U, Meland S, Rosseland BO, et al. Transcriptional changes in Atlantic salmon (Salmo salar) after embryonic exposure to road salt. Aquat Toxicol. 2015;169:58-68. https://doi.org/10.10 6/j.aquatox.2015.10.003.

60. MacRae TH. Stress tolerance during diapause and quiescence of the brine shrimp, Artemia. Cell Stress Chaperones. 2016;21(1):9-18. https://doi.org/10.1 007/s12192-015-0635-7.

61. Hand SC, Menze MA, Toner M, Boswell L, Moore D. LEA proteins during water stress: not just for plants anymore. Annu Rev Physiol. 2011;73(1):11534. https://doi.org/10.1146/annurev-physiol-012110-142203.

62. Janis B, Uversky VN, Menze MA. Potential functions of LEA proteins from the brine shrimp Artemia franciscana - anhydrobiosis meets bioinformatics. $J$ Biomol Struct Dyn. 2018;36(12):3291-309. https://doi.org/10.1080/07391102.2 017.1387177.

63. Nino-Gonzalez M, Novo-Uzal E, Richardson DN, Barros PM, Duque P. More transporters, more substrates: the Arabidopsis major facilitator superfamily revisited. Mol Plant. 2019;12(9):1182-202. https://doi.org/10.1 016/j.molp.2019.07.003.

64. Dung NV, Christiaens O, Le Van Bao D, De Vos S, MacRae TH, Smagghe G, et al. Identification of RNAi-related genes and transgenerational efficiency of RNAi in Artemia franciscana. Aquaculture. 2019;501:285-92.

65. Blagosklonny MV. Hypoxia, MTOR and autophagy: converging on senescence or quiescence. Autophagy. 2013;9(2):260-2. https://doi.org/10.41 61/auto.22783.

66. Bieberich E. Synthesis, processing, and function of N-glycans in Nglycoproteins. Adv Neurobiol. 2014;9:47-70. https://doi.org/10.1007/978-1-4 939-1154-7_3.
67. Scanlon SE, Glazer PM. Multifaceted control of DNA repair pathways by the hypoxic tumor microenvironment. DNA Repair. 2015;32:180-9. https://doi. org/10.1016/j.dnarep.2015.04.030.

68. Mueller CA, Willis E, Burggren WW. Salt sensitivity of the morphometry of Artemia franciscana during development: a demonstration of 3D critical windows. J Exp Biol. 2016;219(Pt 4):571-81. https://doi.org/10.1242/jeb.12 5823

69. Kuballa AV, Holton TA, Paterson B, Elizur A. Moult cycle specific differential gene expression profiling of the crab Portunus pelagicus. BMC Genomics. 2011;12(1):147. https://doi.org/10.1186/1471-2164-12-147.

70. LV J, Zhang L, Liu P, Li J. Transcriptomic variation of eyestalk reveals the genes and biological processes associated with molting in Portunus trituberculatus. PLoS One. 2017;12(4):e0175315. https://doi.org/10.1371/ journal.pone.0175315.

71. Seear PJ, Tarling GA, Burns G, Goodall-Copestake WP, Gaten E, Ozkaya O, et al. Differential gene expression during the moult cycle of Antarctic krill (Euphausia superba). BMC Genomics. 2010;11(1):582. https://doi.org/10.11 86/1471-2164-11-582.

72. Rocha J, Garcia-Carreño FL, Muhlia-Almazán A, Peregrino-Uriarte AB, YépizPlascencia G, Córdova-Murueta JH. Cuticular chitin synthase and chitinase mRNA of whiteleg shrimp Litopenaeus vannamei during the molting cycle. Aquaculture. 2012;330-333(1):111-5.

73. Villarino GH, Bombarely A, Giovannoni JJ, Scanlon MJ, Mattson NS. Transcriptomic analysis of Petunia hybrida in response to salt stress using high throughput RNA sequencing. PloS one. 2014;9(4):e94651.

74. Tuteja N, Tran NQ, Dang HQ, Tuteja R. Plant MCM proteins: role in DNA replication and beyond. Plant Mol Biol. 2011;77(6):537-45. https://doi.org/1 0.1007/s11103-011-9836-3.

75. Flowers TJ, Glenn EP, Volkov V. Could vesicular transport of $\mathrm{Na}+$ and $\mathrm{Cl}-$ be a feature of salt tolerance in halophytes? Ann Botany. 2019;123(1):1-8.

76. Lowy RJ, Conte FP. Isolation and functional characterization of crustacean larval salt gland. Am J Phys. 1985;248(6 Pt 2):R702-8.

77. Hossain MS, Dietz KJ. Tuning of redox regulatory mechanisms, reactive oxygen species and redox homeostasis under salinity stress. Front Plant Sci. 2016;7:548,

78. Rivera-Ingraham GA, Nommick A, Blondeau-Bidet E, Ladurner P, Lignot JH. Salinity stress from the perspective of the energy-redox axis: lessons from a marine intertidal flatworm. Redox Biol. 2016;10:53-64. https://doi.org/10.101 6/j.redox.2016.09.012.

79. Rubio MC, Bustos-Sanmamed P, Clemente MR, Becana M. Effects of salt stress on the expression of antioxidant genes and proteins in the model legume Lotus japonicus. New Phytol. 2009;181(4):851-9. https://doi.org/1 0.1111/j.1469-8137.2008.02718.x.

80. Rodrigo-Moreno A, Poschenrieder C, Shabala S. Transition metals: a double edge sward in ROS generation and signaling. Plant Signal Behav. 2013;8(3): e23425. https://doi.org/10.4161/psb.23425.

81. Tolleter D, Hincha DK, Macherel D. A mitochondrial late embryogenesis abundant protein stabilizes model membranes in the dry state. Biochim Biophys Acta. 2010; 1798(10):1926-33. https://doi.org/10.1016/j.bbamem.2010.06.029.

82. Tanaka S, Tanaka J, Miwa Y, Horikawa DD, Katayama T, Arakawa K, et al. Novel mitochondria-targeted heat-soluble proteins identified in the anhydrobiotic tardigrade improve osmotic tolerance of human cells. PLoS One. 2015;10(2):e0118272. https://doi.org/10.1371/journal.pone.0118272.

83. Yamaguchi A, Tanaka S, Yamaguchi S, Kuwahara H, Takamura C, ImajohOhmi S, et al. Two novel heat-soluble protein families abundantly expressed in an anhydrobiotic tardigrade. PLoS One. 2012;7(8):e44209. https://doi.org/1 0.1371/journal.pone.0044209.

84. Kamilari M, Jørgensen A, Schiøtt M, Møbjerg N. Comparative transcriptomics suggest unique molecular adaptations within tardigrade lineages. BMC Genomics. 2019;20(607):1-9.

85. Okamura YMT, Elshopakey GE, Itami T. Molecular characterization and gene expression analysis of hypoxia-inducible factor and its inhibitory factors in kuruma shrimp Marsupenaeus japonicus. Fish Shellfish Immunol. 2018;79: 168-74. https://doi.org/10.1016/j.fsi.2018.05.015.

86. Graham AM, Barreto FS. Loss of the HIF pathway in a widely distributed intertidal crustacean, the copepod Tigriopus californicus. Proc Natl Acad Sci U S A. 2019;116(26):12913-8. https://doi.org/10.1073/pnas.1819874116.

87. Cowan KJ, Storey KB. Protein kinase and phosphatase responses to anoxia in crayfish, Orconectes virilis: purification and characterization of CAMPdependent protein kinase. Comp Biochem Physiol Part B Biochem Mol Biol. 2001;130(4):565-77. https://doi.org/10.1016/S1096-4959(01)00467-5. 
88. Lant BA. The anoxia resistant crayfish: a profile of stress response. Ottawa: Carleton University; 2011.

89. Fuhrmann DC, Brune B. Mitochondrial composition and function under the control of hypoxia. Redox Biol. 2017;12:208-15. https://doi.org/10.1016/j. redox.2017.02.012

90. Selvy PE, Lavieri RR, Lindsley CW, Brown HA. Phospholipase D: enzymology, functionality, and chemical modulation. Chem Rev. 2011;111(10):6064-119. https://doi.org/10.1021/cr200296t.

91. Bargmann BO, Munnik T. The role of phospholipase D in plant stress responses. Curr Opin Plant Biol. 2006;9(5):515-22. https://doi.org/10.1016/j. pbi.2006.07.011.

92. Baruah K, Phong HP, Norouzitallab P, Defoirdt T, Bossier P. The gnotobiotic brine shrimp (Artemia franciscana) model system reveals that the phenolic compound pyrogallol protects against infection through its prooxidant activity. Free Radic Biol Med. 2015;89(12):593-601. https://doi.org/10.1016/j. freeradbiomed.2015.10.397

93. Norouzitallab PBK, Biswas P, Vanrompay D, Bossier P. Probing the phenomenon of trained immunity in invertebrates during a transgenerational study, using brine shrimp Artemia as a model system. Sci Rep. 2016;6(21166):1-4.

94. Zhang X, Yuan J, Sun Y, Li S, Gao Y, Yu Y, et al. Penaeid shrimp genome provides insights into benthic adaptation and frequent molting. Nat Commun. 2019;10(356):1-4

95. Nambu F, Tanaka S, Nambu Z. Inbred strains of brine shrimp derived from Artemia franciscana: lineage, RAPD analysis, life span, reproductive traits and mode, adaptation, and tolerance to salinity changes. Zool Sci. 2007;24(2): 159-71. https://doi.org/10.2108/zsj.24.159.

96. Coutteau P, Brendonck L, Lavens P, Sorgeloos P. The use of manipulated baker's yeast as an algal substitute for the laboratory culture of Anostraca. Hydrobiologia. 1992;234(1):25-32. https://doi.org/10.1007/BF00010776.

97. Hodgson R. CTAB method for the isolation of total nucleic acid (TNA) from shrimp tissue. In: Workshop on "Molecular diagnostics for shrimp viruses in the Asian region". February 10-13. Salaya: Mahidol University; 1999.

98. Andrews S. FastQC: a quality control tool for high throughput sequence data; 2010.

99. csf-ngs/fastqc. 2012 [cited March 2016]. Available from: https:/github.com/ csf-ngs/fastqc/blob/master/Contaminants/contaminant_list.txt. Accessed 2016.

100. CLC bio. White paper on de novo assembly in CLC Assembly Cell 4.0. 2012.

101. Salmela L, Rivals E. LoRDEC: accurate and efficient long read error correction. Bioinformatics. 2014;30(24):3506-14. https://doi.org/10.1093/ bioinformatics/btu538.

102. Van Nieuwerburgh F, Thompson RC, Ledesma J, Deforce D, Gaasterland T, Ordoukhanian $\mathrm{P}$, et al. Illumina mate-paired DNA sequencing-library preparation using Cre-Lox recombination. Nucleic Acids Res. 2012;40(3):e24.

103. Bankevich A, Nurk S, Antipov D, Gurevich AA, Dvorkin M, Kulikov AS, et al. SPAdes: a new genome assembly algorithm and its applications to single-cell sequencing. J Comp Biol. 2012;19(5):455-77. https:/doi.org/10.1089/cmb.2012.0021.

104. Ye CX, Hill CM, Wu SG, Ruan J, Ma ZS. DBG2OLC: efficient assembly of large genomes using Long erroneous reads of the third generation sequencing technologies. Sci Rep. 2016;6:1-9.

105. Xue W, Li JT, Zhu YP, Hou GY, Kong XF, Kuang YY, et al. L_RNA_scaffolder: scaffolding genomes with transcripts. BMC Genomics. 2013;14(1). https://doi. org/10.1186/1471-2164-14-604.

106. Boetzer M, Henkel CV, Jansen HJ, Butler D, Pirovano W. Scaffolding preassembled contigs using SSPACE. Bioinformatics. 2011;27(4):578-9. https:// doi.org/10.1093/bioinformatics/btq683.

107. Boetzer M, Pirovano W. Toward almost closed genomes with GapFiller. Genome Biol. 2012;13(6):R56. https://doi.org/10.1186/gb-2012-13-6-r56.

108. Li H, Durbin R. Fast and accurate short read alignment with burrowswheeler transform. Bioinformatics. 2009;25(14):1754-60. https://doi.org/10.1 093/bioinformatics/btp324.

109. Walker BJ, Abeel T, Shea T, Priest M, Abouelliel A, Sakthikumar S, et al. Pilon: An integrated tool for comprehensive microbial variant detection and genome assembly improvement. PloS one. 2014;9(11):e112963.

110. Heydari M, Miclotte G, Van de Peer Y, Fostier J. BrownieAligner: accurate alignment of Illumina sequencing data to de Bruijn graphs. BMC Bioinformatics. 2018;19(1):311. https://doi.org/10.1186/s12859-018-2319-7.

111. Bolger AM, Lohse M, Usadel B. Trimmomatic: a flexible trimmer for Illumina sequence data. Bioinformatics. 2014;30(15):2114-20. https://doi.org/10.1093/ bioinformatics/btu170.
112. Bushnell B, Rood J, Singer E. BBMerge - accurate paired shotgun read merging via overlap. PLoS One. 2017;12(10):e0185056. https://doi.org/10.13 71/journal.pone.0185056.

113. Bushmanova E, Antipov D, Lapidus A, Prjibelski AD. rnaSPAdes: a de novo transcriptome assembler and its application to RNA-Seq data. Gigascience. 2019;8(9):giz100. https://doi.org/10.1093/gigascience/giz100.

114. Dobin A, Davis CA, Schlesinger F, Drenkow J, Zaleski C, Jha S, et al. STAR: ultrafast universal RNA-seq aligner. Bioinformatics. 2013;29(1):15-21. https:// doi.org/10.1093/bioinformatics/bts635.

115. Kim D, Paggi JM, Park C, Bennett C, Salzberg SL. Graph-based genome alignment and genotyping with HISAT2 and HISAT-genotype. Nat Biotechnol. 2019;37(8):907-15. https://doi.org/10.1038/s41587-019-0201-4.

116. Degroeve S, Saeys Y, De Baets B, Rouze P, Van de Peer Y. SpliceMachine: predicting splice sites from high-dimensional local context representations. Bioinformatics. 2005;21(8):1332-8. https://doi.org/10.1093/bioinformatics/bti166.

117. Pruitt KD, Tatusova T, Brown GR, Maglott DR. NCBI reference sequences (RefSeq): current status, new features and genome annotation policy. Nucleic Acids Res. 2012;40(D1):D130-D5. https://doi.org/10.1093/nar/gkr1079.

118. Sterck L, Billiau K, Abeel T, Rouze $P$, van de Peer Y. ORCAE: online resource for community annotation of eukaryotes. Nat Methods. 2012;9(11):1041.

119. Abeel T, Van Parys T, Saeys Y, Galagan J, Van de Peer Y. GenomeView: a next-generation genome browser. Nucleic Acids Res. 2012;40(2):e12.

120. Simao FA, Waterhouse RM, loannidis P, Kriventseva EV, Zdobnov EM. BUSCO: assessing genome assembly and annotation completeness with single-copy orthologs. Bioinformatics. 2015;31(19):3210-2. https://doi.org/1 0.1093/bioinformatics/btv351.

121. Bao W, Kojima KK, Kohany O. Repbase update, a database of repetitive elements in eukaryotic genomes. Mob DNA. 2015;6(1):11. https://doi.org/1 0.1186/s13100-015-0041-9.

122. Pearson WR. An introduction to sequence similarity ("homology") searching. Curr Protoc Bioinform. 2013;3:1-3.

123. Refseq release 94. 2019 [cited 06/06/19]. Available from: https://www.ncbi. nlm.nih.gov/refseq/. Accessed 2019.

124. Sun $X Y$, Xia $X H$, Yang Q. Dating the origin of the major lineages of Branchiopoda. Palaeoworld. 2016;25(2):303-17. https://doi.org/10.1016/j.pa Iwor.2015.02.003.

125. Emms DM, Kelly S. OrthoFinder: solving fundamental biases in whole genome comparisons dramatically improves orthogroup inference accuracy. Genome Biol. 2015;16(1):157. https://doi.org/10.1186/s13059-015-0721-2.

126. Heberle H, Meirelles GV, da Silva FR, Telles GP, Minghim R. InteractiVenn: a web-based tool for the analysis of sets through Venn diagrams. BMC Bioinformatics. 2015;16(1):169. https://doi.org/10.1186/s12859-015-0611-3.

127. Untergasser A. RNAprep: Trizol combined with columns 2008. Available from: www.untergasser.de/lab.

128. Ewels P, Magnusson M, Lundin S, Kaller M. MultiQC: summarize analysis results for multiple tools and samples in a single report. Bioinformatics. 2016;32(19):3047-8. https://doi.org/10.1093/bioinformatics/btw354.

129. Liao Y, Smyth GK, Shi W. featureCounts: an efficient general purpose program for assigning sequence reads to genomic features. Bioinformatics. 2014;30(7):923-30. https://doi.org/10.1093/bioinformatics/btt656.

130. Van den Berge K, Soneson C, Robinson MD, Clement L. stageR: a general stagewise method for controlling the gene-level false discovery rate in differential expression and differential transcript usage. Genome Biol. 2017;18(1):151.

131. Robinson MD, McCarthy DJ, Smyth GK. edgeR: a Bioconductor package for differential expression analysis of digital gene expression data. Bioinformatics. 2010;26(1):139-40. https://doi.org/10.1093/bioinformatics/ btp616.

132. Gotz S, Garcia-Gomez JM, Terol J, Williams TD, Nagaraj SH, Nueda MJ, et al. Highthroughput functional annotation and data mining with the Blast2GO suite. Nucleic Acids Res. 2008;36(10):3420-35. https://doi.org/10.1093/nar/gkn176.

133. Szklarczyk D, Franceschini A, Wyder S, Forslund K, Heller D, Huerta-Cepas J, et al. STRING v10: protein-protein interaction networks, integrated over the tree of life. Nucleic Acids Res. 2015;43(Database issue):D447-52. https://doi. org/10.1093/nar/gku1003.

134. KEGG Mapper - Search\&Color Pathway. 2019.

\section{Publisher's Note}

Springer Nature remains neutral with regard to jurisdictional claims in published maps and institutional affiliations. 